\title{
Analytical modeling of cognitive heterogeneous cellular networks over Nakagami-m fading
}

\author{
Fereidoun H Panahi ${ }^{*}$ and Tomoaki Ohtsuki
}

\begin{abstract}
In this paper, we present a cognitive radio (CR)-based statistical framework for a two-tier heterogeneous cellular network (femto-macro network) to model the outage probability at any arbitrary secondary (femto) and primary (macro) user. A system model based on stochastic geometry (utilizing the spatial Poisson point process (PPP) theory) is applied to model the random locations and network topology of both secondary and primary users. A considerable performance improvement can be generally achieved by mitigating interference in result of applying the CR idea over the above model. Novel closed-form expressions are derived for the outage probability of any typical femto and macro user considering the Nakagami-m fading for each desired and interference links. We also study some important design factors which their important role in the determination of outage and interference cannot be ignored. We conduct simulations to validate our analytical results and evaluate the proposed schemes in terms of outage probability for different values of signal-to-interference-plus-noise-ratio (SINR) target.
\end{abstract}

Keywords: Stochastic geometry; Cognitive radio; Outage probability

\section{Introduction}

The best solution to the spectrum saturation and bandwidth availability problems in multi-tier cellular networks is to adopt technologies that make the most efficient use of existing spectrum through frequency reuse schemes $[1,2]$. In universal frequency reuse scheme, the existing spectrum can be aggressively and effectively reused by all of the coexisting network tiers. This will lead to higher spatial spectrum utilization and network usage capacity at the expense of an increased possibility of interference among network tiers and of a reduced quality of service (QoS). In multi-tier cellular networks, interference is increasingly becoming a major performancelimiting factor, and hence, interference modeling, coordination, and avoidance are the primary focus of interest for both the industry and academic communities. Applying the cognitive radio (CR) technology in multi-tier cellular networks to be aware of and adapt to communication environments, some of the above challenges can be tackled. In fact, $\mathrm{CR}$ is the key enabling technology for interference management and avoidance in multi-tier cellular networks $[2,3]$.

\footnotetext{
* Correspondence: fereidoun@ohtsuki.ics.keio.ac.jp

Graduate School of Science and Technology, Keio University, 3-14-1 Hiyoshi,
} Kouhoku-ku, 223-8522 Yokohama, Japan
On the other hand, the aggregate interference environment is more complicated to model, and evaluating the performance of communication techniques in the presence of heterogeneous interference is challenging. For interference characterization, if the base stations (BSs) of the cellular network follow a regular grid (e.g., the traditional hexagonal grid model), then the signal-tointerference-plus-noise-ratio (SINR) characterization will be either intractable $[3,4]$ or inaccurate due to unrealistic assumptions [5]. Moreover, as urban areas are built out, the BS infrastructure is becoming less like points on a hexagonal lattice and more random. Hence, the use of a hexagonal grid to model the BS locations is violated and is considered too idealized [6]. Furthermore, according to [4] and [6] for snapshots of a cellular network at different locations, the positions of the BSs with respect to each other follow random patterns due to the size and unpredictability of the BSs in these kind of networks. Therefore, the need for a powerful mathematical and statistical tool for modeling, analysis, and design of wireless networks with random topologies is quite obvious.

A new modeling approach called 'stochastic geometry' has been recently applied to the analysis of multi-tier cellular networks due to its ability to capture the topological randomness in the network and its aim at 
deriving accurate and tractable expressions for outage probability $[3,6]$. Stochastic geometry stems from applied probability and has a wide range of applications in the analysis and design of wireless networks in particular for modeling and analyzing systems with random channel access (e.g., ALOHA $[7,8]$ and carrier sensing multiple access (CSMA) [9]), single- and multi-tier cellular networks [6], and networks with cognitive abilities $[7,10]$. This paper discusses this new theoretical model to provide a better understanding of the heterogeneous cellular networks of tomorrow and their challenges (interference modeling, coordination, and avoidance) that must be tackled in order for these networks to reach their potential. We focus on a two-tier femto-macro network where low-power and small-coverage local nodes (femto nodes) are distributed in the coverage of macro nodes. We provide an insight into the role of $\mathrm{CR}$ in interference mitigation in two-tier heterogeneous networks. We derive closed-form expressions for the outage probability of any typical femto and macro user in the network. We also study the effect of several important design factors which play vital roles in the determination of outage and interference.

The main contributions are therefore the following: (1) We analyze the Laplace transforms of all four types of aggregate interference between macro and CR femto networks (including the interference between macro nodes among themselves and femto nodes among themselves, the cross-interference from femto to macro network and vice versa) in perfect and imperfect spectrum sensing CR-based femto networks, considering simultaneously the Nakagami fading, the Poisson point process (PPP) model, and some important design factors which play vital roles in determination and mitigation of outage and interference. (2) This article provides an insight into the role of $\mathrm{CR}$ in interference mitigation in orthogonal frequency-division multiple-access (OFDMA) two-tier heterogeneous networks. (3) Tight closed-form expressions are derived for the outage probability of any typical femto and macro user considering the Nakagami fading (Rayleigh fading assumption is used in many studies to relax the difficulty of addressing a closed-form expression for the outage probability (see, e.g., [8] and [10])) for all the communication links (desired and interference links) with the possibility of using the $\mathrm{CR}$ ability for the femto network.

To the best of our knowledge, no closed-form expressions for the outage probability of the femto and macro users exist in the current literature without imposing the Rayleigh fading assumption. On the other hand, most of the available studies in this area are based on the existence of only one macro-BS (along with the macro users and the femto network) and the effect of considering multiple macro-BSs is ignored in the analysis of outage probability.

\section{System model}

\subsection{Model description}

We consider infinite spatially collocated macro-BS and femto-BS node heterogeneous networks (see Figure 1). It is assumed that the spatial distribution of the nodes is captured using two collocated and independent homogenous Poisson point processes (HPPPs) [6,7] i.e., $\Phi_{M}$ and $\Phi_{F}$ with intensities $\lambda_{M}$ and $\lambda_{F}$, respectively. In other words, the locations of the macro-BS nodes constitute an HPPP $\Phi_{M}$, where $\lambda_{M}$ is the average number of the macro-BS nodes per unit area. Similar statement can be made for the HPPP formed by the femto-BS nodes $\Phi_{F}$ with intensity $\lambda_{F}$. According to superposition theorem [11], the overall node process over the network formed by both the macroBS and femto-BS nodes also is an HPPP with intensity $\lambda\left(\lambda=\lambda_{F}+\lambda_{M}\right)$. Furthermore, the macro and femto users are scattered over the plane according to some independent PPPs with different densities than $\lambda_{M}$ and $\lambda_{F}$, respectively. However, our interference analysis is fundamentally concerned with the distribution of the transmitters (BSs).

Since femto-BSs are installed and maintained by the paying home users for better indoor performance, they are only accessible by their own mobile subscribers (femto users) (known as closed-access policy). On the other hand, macro-BSs can be accessed only by unauthorized users (macro users). In practice, macro network is deployed usually without awareness of the distributed femto network. To this end, wireless operators can consider giving priority to the macro users, and the femto network has to be self-optimized to mitigate its interference to the macro users. Motivated by this insight, the macroBSs (along with the macro users) and the CR-enabled femto-BSs (along with the femto users) are analogous to primary and secondary systems in the CR model, respectively.

\subsection{System structure}

In OFDMA, the spectrum is orthogonally divided into time-frequency resource blocks (RBs), which increases flexibility in resource allocation, thereby allowing high spectral efficiency. As shown in Figure 2, we consider a spectrum of $N$ RBs, out of which $M(M \leq N)$ random RBs are idle or unoccupied by the macro users (primary system). With the CR capability, a femto-BS could actively acquire knowledge about its environment and access to the RBs without the aid of a macrocell in a decentralized fashion (clearly, no synchronization between the macro and femto network is needed any more) and automatically prevent disturbing the macro users [2].

1) As shown in Figure 2, each femto-BS's transmission strategy is divided into consecutive slots, each 


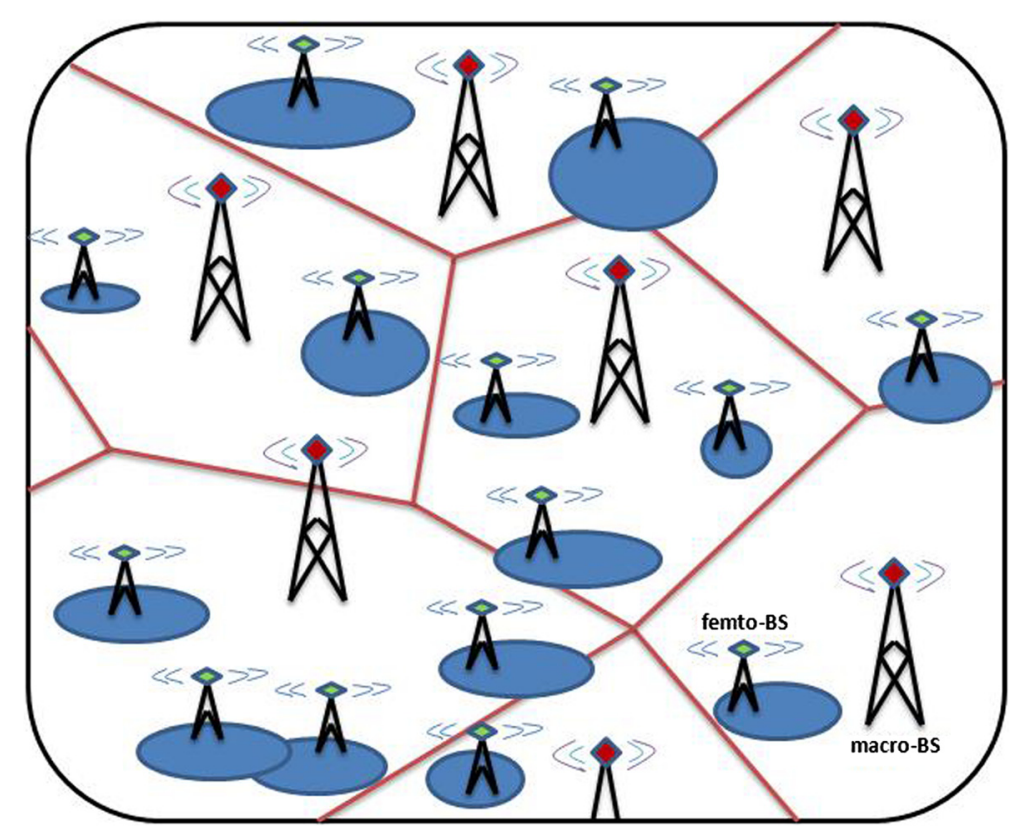

Figure 1 The heterogeneous model (femto and macro BSs).

having a duration of $T$. Each slot is divided into two consecutive stages, i.e., sensing and data transmission, with durations of $T_{S}$ and $T_{D}$, respectively. Each femto-BS periodically senses the spectrum to identify which RBs are occupied by the macro network. Indeed, each femto-BS accomplishes sensing one RB in one unit slot $T_{S R B}$ within $T_{S}$. Each femto-BS senses $N_{s}$ RBs in sequence which are randomly selected from the $N$ available RBs and detects its idle RB set. Clearly, the time required for sensing the $N_{s}$ RBs is $T_{s}=T_{S R B} N_{s}$. Note that the femto-BSs cannot perform data transmission within the sensing time $T_{S}$. We assume that all femto-BSs are perfectly synchronized and have the same time as the sensing time. Methods for implementing a perfect synchronization among the femto-BSs are outside the scope of this paper; however, a set of possible candidates exist, including GPS synchronization, the wired backhaul (IEEE 1588), and leveraging synchronization signals broadcasted by the femto-BSs [12].

2) Each femto-BS senses the received interference power on each RB within the sensing duration.

- If the received interference power on an RB exceeds a certain threshold, the RB is identified as being occupied by the macro network since all femto-BSs sense at the same time.

- Otherwise, the RB is unoccupied by the macro network.

3) In the data transmission time $\left(T_{D}\right)$, each femto-BS only allocates an unoccupied RB sensed in the sensing time to its user (by only utilizing these unoccupied RBs, cross-tier interference can be consequently avoided). Since the determination of each individual RB status as busy/idle is subject to (occasional) error, determined by the probability of (correct) detection of the presence of PUs' signals $P_{d}$

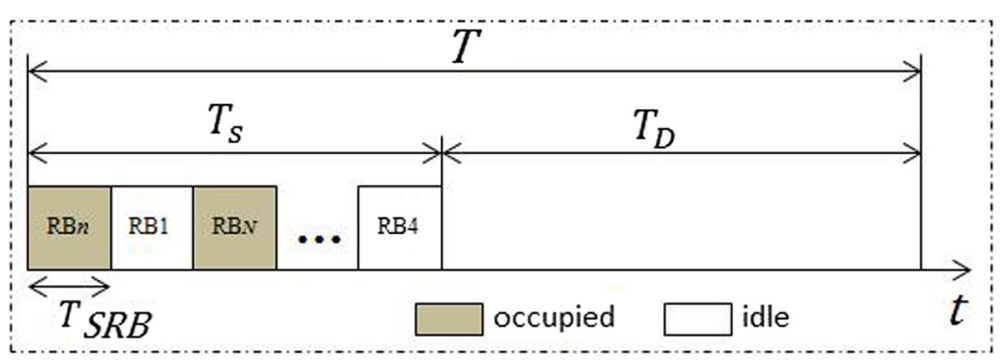

Figure 2 The CR femto-BS's transmission strategy in one time slot. 
and probability of false alarm $P_{f}$ (probability of falsely declaring an idle RB as busy), we study the effect of both the ideal detection, i.e., $P_{d}=1$ and $P_{f}=0$, and the cases involving imperfect sensing, i.e., $P_{d} \neq 1$ and $P_{f} \neq 0$ on the outage probabilities of femto and macro users.

As shown in Figure 3, the macro users' exclusion regions with radius $D$ are used to guarantee that the femto-BSs will, on average, not generate an aggregate interference leading to the outage of macro (primary) users. As shown, for example, those femto-BSs located in the tagged macro user's exclusion region are not allowed to transmit data whether they pick the same RB as the tagged macro user or a different one. The tagged macro user is not disturbed by the femto-BSs transmitting on different RBs (from that of the tagged macro) even if they are inside its exclusion region. However, we deactivate them to prevent the tagged macro user from any harmful interference as a result of sudden appearance of them on the same RB in next time slots. We consider this law throughout the paper even in the case of the perfect sensing scenario. We also assume that the femto users are informed about the macro users' exclusion regions.

\section{Stochastic geometry-based network configuration}

\subsection{Femto outage probability}

We derive the probability of outage for a typical femto user $\left(p_{O F}\right)$ defined as the probability that a randomly chosen femto user cannot achieve a target SINR $\theta$ considering a collocated spectrum sensing CR based femto network and macro-BSs as follows [6]:

$$
p_{\mathrm{OF}}=1-P[\operatorname{SINR}>\theta]
$$

In fact, the outage probability evaluates the cumulative distribution function (CDF) of SINR over the entire network. The experienced SINR by a typical femto user is calculated as

$$
\operatorname{SINR}=\frac{P_{F} h_{F} r_{F}^{-\alpha}}{\sigma^{2}+I_{F B}+I_{M B}}
$$

where $P_{F}$ is the transmission power from the nearest femto-BS (tagged femto-BS) located in the random distance $r_{F}$ from its tagged femto user (we assume that the tagged femto user under consideration is located at the origin), and $\alpha$ is the path-loss exponent. $I_{F B}$ and $I_{M B}$ are the aggregate interference power at the origin from the other femto-BSs and macro-BSs, respectively, and $\sigma^{2}$ is the noise power.

When the fading distribution is Nakagami- $m_{d, F}$ for the communication channel between the tagged femto user and its corresponding femto-BS, the probability density function (pdf) of the power fading coefficient $x=h_{F}$ is as follows

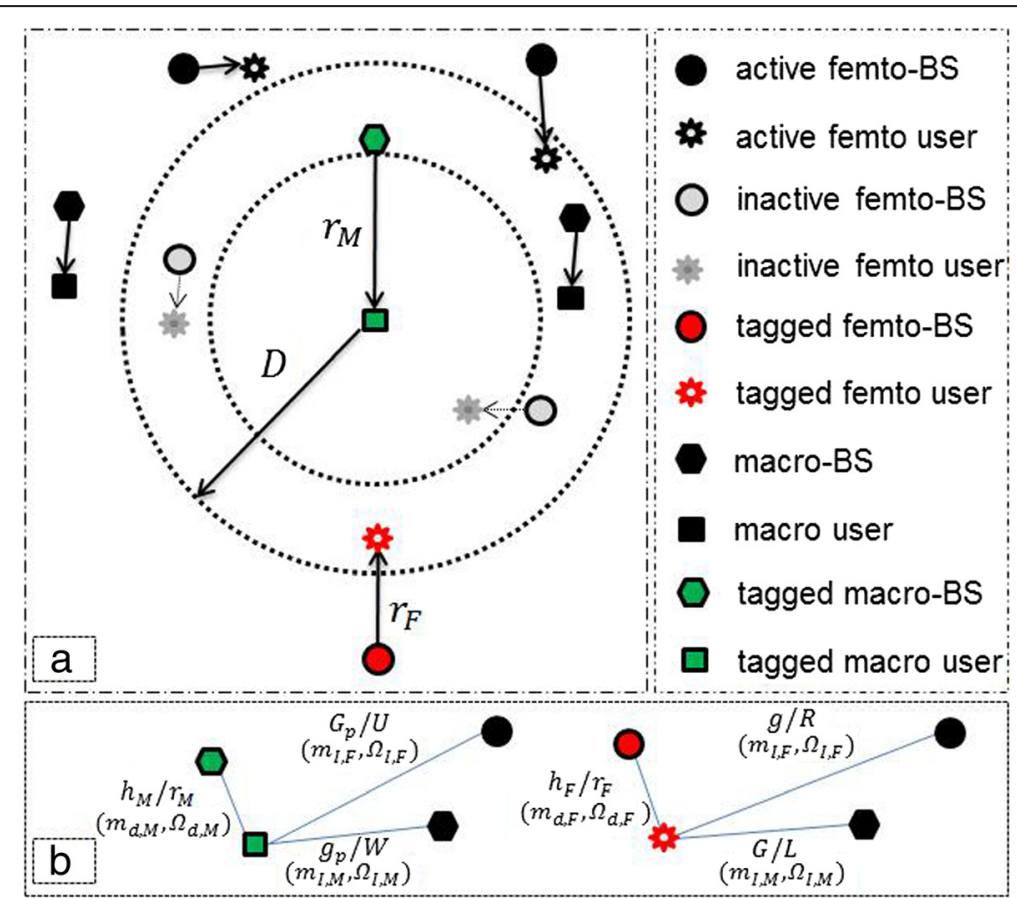

Figure 3 The macro users' exclusion regions. (a) The bipolar network model. (b) The link gains/distances, and Nakagami parameters. 


$$
p(x)=\frac{m_{d, F} m_{d, F}}{\Omega_{d, F} m_{d, F} \Gamma\left(m_{d, F}\right)}(x)^{m_{d, F}-1} e^{-\frac{m_{d, F} x}{\Omega_{d, F}}}
$$

where $m_{d, F}$ describes the severity of the fading for the desired link, and $\Omega_{d, F}$ is the average SNR. By changing $m_{d, F}$ we can get a variety of fading conditions. For example, if $m_{d, F}=1$, then the desired link suffers from Rayleigh fading, and $m_{d, F}=\infty$ means that there is no fading. Note that we will represent the Nakagami fading parameters for each communication link, separately (see Figure $3 \mathrm{~b}$ ). Even though the fading power gain distributions are identical, we will still use different symbols to distinguish all the existing links for the sake of analytical convenience.

Here, we derive the complementary CDF (CCDF) of SINR as follows

$$
\begin{gathered}
P[\text { SINR }>\theta]=P\left[\frac{P_{F} h_{F} r_{F}^{-\alpha}}{\sigma^{2}+I_{F B}+I_{M B}}>\theta\right] \\
=P\left[h_{F}>\left(\sigma^{2}+I_{F B}+I_{M B}\right) \frac{\theta r_{F}^{\alpha}}{P_{F}}\right] \\
=\mathrm{E}_{I}\left[P\left[h_{F}>(I) \frac{\theta r_{F}^{\alpha}}{P_{F}}\right]\right] .
\end{gathered}
$$

Considering the pdf of the power fading coefficient $h_{F}$ and the incomplete gamma function (Note that $\Gamma(a, x)=\int_{x}^{\infty} t^{a-1} e^{-t} \mathrm{~d} t$ indicates the incomplete gamma function), we have

$$
\begin{gathered}
P\left[h_{F}>(I) \frac{\theta r_{F}^{\alpha}}{P_{F}}\right]=\int_{\frac{\theta r_{F}^{\alpha}}{P_{F}}(I)}^{\infty} p(x) \mathrm{d} x \\
=\frac{-m_{d, F} m_{d, F}(-1)^{m_{d, F}-1}}{\left(-m_{d, F}\right)^{m_{d, F}}}\left[\frac{\Gamma\left[m_{d, F}, \frac{m_{d, F}}{\Omega_{d, F}}\left(\frac{\theta r_{F}^{\alpha}}{P_{F}}(I)\right)\right]}{\Gamma\left(m_{d, F}\right)}\right]
\end{gathered}
$$

where $I=\sigma^{2}+I_{F B}+I_{M B}$.

We know that $\frac{\Gamma[m, m y]}{\Gamma(m)}=e^{-m y} \sum_{k=0}^{m-1} \frac{m^{k}}{k !} y^{k}$ ( $m$ is a positive integer). Therefore, $P\left[h_{F}>(I) \frac{\theta r_{F}{ }^{\alpha}}{P_{F}}\right]$ is simplified as follows

$$
\left[e^{-\frac{m_{d, F}}{\Omega_{d, F}}\left(\frac{\theta r_{F}{ }^{\alpha}}{P_{F}}\right) I} \sum_{k=0}^{m_{d, F}-1} \frac{\left(\frac{m_{d, F}}{\Omega_{d, F}} \frac{\theta r_{F}{ }^{\alpha}}{P_{F}}\right)^{k}}{k !} I^{k}\right]
$$

From (3) and (4) we have

$$
P[\operatorname{SINR}>\theta]=\int_{0}^{\infty}\left[e^{-\frac{m_{d, F}}{\Omega_{d, F}}\left(\frac{\theta r_{F}{ }^{\alpha}}{P_{F}}\right)} I^{m_{d, F}-1} \sum_{k=0}^{\frac{\left(\frac{m_{d, F} \theta r_{F}^{\alpha}}{\Omega_{d, F} P_{F}}\right)^{k}}{k !}} I^{k}\right] f_{I}(i) \mathrm{d} i .
$$

Finally, the CCDF of SINR when the fading distribution is Nakagami- $m_{d, F}$ for the communication channel between the tagged femto user and its corresponding femto-BS is given by

$$
P[\operatorname{SINR}>\theta]=\left[\sum_{k=0}^{m_{d, F}-1} \frac{(s)^{k}}{k !}(-1)^{k} \frac{d^{k} \mathcal{L}_{I}(s)}{d s^{k}}\right]
$$

where $I=\sigma^{2}+I_{F B}+I_{M B}$ and $s=\frac{m_{d, F} \theta r_{F}{ }^{\alpha}}{\Omega_{d, F} P_{F}}$.

Proof: See Appendix 1.

Note that due to the assumption of independent PPPs for the femto and macro networks, the aggregate interference received from the femto-BSs is independent of the aggregate interference received from the macro-BSs [10]; therefore, we can write

$$
\begin{aligned}
& \mathcal{L}_{I}(s)=F_{I}(s)=\mathrm{E}\left[e^{-s I}\right] \\
& =\mathrm{E}\left[e^{-s\left(\sigma^{2}+I_{F B}+I_{M B}\right)}\right] \\
& =\mathrm{E}\left[e^{-s I_{F B}}\right] \mathrm{E}\left[e^{-s I_{M B}}\right] \mathrm{E}\left[e^{-s \sigma^{2}}\right] \\
& =\mathcal{L}_{I_{F B}}(s) \mathcal{L}_{I_{M B}}(s) e^{-s \sigma^{2}}
\end{aligned}
$$

Where $\mathcal{L}_{I_{F B}}(s)$ and $\mathcal{L}_{I_{M B}}(s)$ are the Laplace transform of random variables $I_{F B}$ and $I_{M B}$ evaluated at $s\left(s=\frac{m_{d, F} \theta r_{F}^{\alpha}}{\Omega_{d, F} P_{F}}\right)$ respectively. Therefore, (6) can be rewritten as follows

$$
P[\operatorname{SINR}>\theta]=\left[\sum_{k=0}^{m_{d, F}-1} \frac{(s)^{k}}{k !}(-1)^{k} \frac{d^{k}\left(\mathcal{L}_{I_{F B}}(s) \mathcal{L}_{I_{M B}}(s) e^{-s \sigma^{2}}\right)}{d s^{k}}\right] .
$$

\subsubsection{Computation of $\mathcal{L}_{I_{F B}}(s)$ and $\mathcal{L}_{I_{M B}}(s)$ at the tagged} femto user

We now calculate the closed-form expressions for $\mathcal{L}_{I_{F B}}$ $(s)$ and $\mathcal{L}_{I_{M B}}(s)$ (at $s=\frac{m_{d, F} \theta r_{F}{ }^{\alpha}}{\Omega_{d, F} P_{F}}$ ). The Laplace transform of the aggregate interference (from all the active femto-BSs except the tagged femto-BS denoted by $\left.f b s_{0}\right)$ is given as follows (Note: some of the femto-BSs located in the macro users' exclusion regions (with radius $D$ ) are deactivated, therefore, $\lambda_{F}^{\prime} \leq \lambda_{F}$ (see Figure 3)) 


$$
\begin{aligned}
\mathcal{L}_{I_{F B}}(s) & =\mathrm{E}_{I_{F B}}\left[\exp \left(-s I_{F B}\right)\right] \\
& =\mathrm{E}_{\Phi_{F}, g_{i}}\left[\exp \left(-s \sum_{i \in \Phi_{F} \backslash\left\{f b s_{0}\right\}} P_{F} g_{i} R_{i}^{-\alpha}\right)\right] \\
& =\mathrm{E}_{\Phi_{F}}\left[\prod_{i \in \Phi_{F} \backslash\left\{f b s_{0}\right\}} \mathrm{E}_{g_{i}}\left[\exp \left(-s P_{F} g_{i} R_{i}^{-\alpha}\right)\right]\right]
\end{aligned}
$$

where $R_{i}$ is the distance of the $i$ th interferer from the tagged femto receiver captured by the point process $\Phi_{F}$. The interference channel gains $g_{i}$ are assumed to be mutually independent and have identical pdfs (Nakagami- $m_{I, F}$ ). Each of the active interfering femto-BSs transmits with the same power $P_{F}$. Using the definition of the Generating functional [6] for the PPP, which states for some func-

tion $f(x)$ that $\mathrm{E}\left[\prod_{x \in \Phi} f(x)\right]=\exp \left(-\int_{\mathbb{R}^{d}}(1-f(x)) \lambda \mathrm{d} x\right)$,

can be rewritten as

$$
\mathcal{L}_{I_{F B}}(s)=\exp \left\{-\mathrm{E}_{g}\left[\int_{r_{F}}^{\infty}\left(1-\exp \left(-s P_{F} g R^{-\alpha}\right)\right) \lambda_{I}(R) \mathrm{d} R\right]\right\}
$$

where we flipped the order of integration and expectation. Since the closest interfering femto-BS is at least at distance $r_{F}$ from the tagged user, the integration limits are from $r_{F}$ to $\infty$. In other words, interference is encountered from all the active femto-BSs located in the area $\mathbb{R}^{d} b\left(0, r_{F}\right)$ (where $b(x, y)$ is ball of radius $y$ centered at point $x$ ). Therefore, not all the femto-BSs will contribute towards the aggregate interference, i.e., only those active femto-BSs which are outside the mentioned ball and at minimum satisfy all of the following conditions qualify as potential contributors.

Before explaining the following conditions ((a) and (b)), it is useful to translate the point process into polar coordinates. Therefore, according to [7] and [11] the intensity of the HPPP $\Phi_{F}$ is shown as

$$
\lambda_{I}(R)=\lambda_{F}^{\prime} d R^{d-1} b_{d}
$$

where $R$ is the distance between an arbitrary femto-BS and the tagged femto receiver. $b_{d}$ is the volume of a unit sphere in $\mathbb{R}^{d}\left(b_{d}=\frac{\sqrt{\pi^{d}}}{\Gamma(1+d / 2)}, \Gamma(x)=\int_{0}^{\infty} t^{x-1} e^{-t} \mathrm{~d} t\right.$ denotes the standard gamma function).

(a) Satisfying the aforementioned condition, any active femto-BS contributes towards the interference at the tagged femto receiver, if it picks the same RB as the tagged femto-BS to communicate with its user, (we show the probability of picking a same RB from a pool of all RBs as $p_{R B}$ (the calculation of $p_{R B}$ for this case is derived in Section 4, Scenario I, Case 1)). (b) We assume that the CR femto-BSs employ a slotted ALOHA MAC (medium access control) protocol to schedule their transmission. Therefore, they only transmit with probability $p_{t x}$ in the current time slot and defer the transmission with probability $1-p_{t x}$.

Applying both conditions (a) and (b) which are indeed two independent thinning tools, the thinned point process of $\Phi_{F}$ is formed. In other words, $\Phi_{F}$ is subjected to two thinning processes each presenting an independent deletion/retention of each point of $\Phi_{F}$ with a fixed retention probability. We now formulize this operation, which will in fact result in reducing the number of interferers and hence lowering the outage probability at the tagged femto user. First, $\Phi_{F}$ is reconstructed by $p_{R B}$-thinning, where each point of $\Phi_{F}$ is retained with probability $p_{R B}$ (to accommodate condition (a)), and then it is thinned again by applying the second independent thinning (to accommodate condition (b)). Thus, the intensity of the process (the number of interfering CR femtoBSs) becomes

$$
\lambda_{I}(R)=\lambda_{F}^{\prime} d R^{d-1} b_{d} p_{R B} p_{t x}
$$

Now, (10) is rewritten as

$$
\exp \left\{-\mathrm{E}_{g}\left[\int_{r_{F}}^{\infty}\left(1-\exp \left(-s P_{F} g R^{-\alpha}\right)\right) \lambda_{F}^{\prime} b_{d} p_{R B} p_{t x} d R^{d-1} \mathrm{~d} R\right]\right\} .
$$

By changing the variables $R^{d} \rightarrow x$ and then $x^{\frac{\alpha}{d}} \rightarrow y$, and following a few simple steps, the above expression is simplified as:

$$
\mathcal{L}_{I_{F B}}(s)_{\mid s=\frac{m_{d, F}, \theta_{r} \alpha}{\Omega_{d, F} F_{F}}}=e^{r_{F}^{d} b_{d} p_{R B} p_{t x} \lambda_{F}^{\prime}-\frac{d}{\alpha} b_{d} p_{R B} p_{t x} \lambda_{F}^{\prime}\left(\frac{m_{d, F}}{\Omega_{d, F}} \theta\right)^{\frac{d}{\alpha}} r_{F}^{d} M(\theta, \alpha)}
$$

where

$$
M(\theta, \alpha)=\mathrm{E}\left[(g)^{\frac{d}{\alpha}}\left(\Gamma\left(-\frac{d}{\alpha}, \frac{m_{d, F}}{\Omega_{d, F}} \theta g\right)-\Gamma\left(-\frac{d}{\alpha}\right)\right)\right] .
$$

Similar with the desired link, we consider the Nakagami power fading model for the femto interfering links as well (Nakagami fading links with equal parameters $m_{I, F}$ and $\Omega_{I, F}$ ). Then, following the derivations in Appendix 2, $M(\theta, \alpha)$ is expressed as 


$$
\begin{aligned}
& M(\theta, \alpha)=\left(\frac{-\Gamma\left(-\frac{d}{\alpha}\right) m_{I, F} m_{I, F}\left(\frac{m_{d, F}}{\Omega_{d, F}} \theta\right)^{-\frac{d}{\alpha}}}{\Omega_{I, F} m_{I, F} \Gamma\left(m_{I, F}\right)}\right) \\
& \times\left[\sum_{k=0}^{\infty} \frac{\left(\frac{m_{d, F}}{\Omega_{d, F}} \theta\right)^{k}\left(\frac{\Omega_{I, F} \Omega_{d, F}}{m_{I, F} \Omega_{d, F}+\Omega_{I, F} m_{d, F} \theta}\right)^{m_{I, F}+k}}{\Gamma\left(k+1-\frac{d}{\alpha}\right)} \Gamma\left(m_{I, F}+k\right)\right] .
\end{aligned}
$$

Using the similar approach, the Laplace transform of the aggregate interference generated by the macro-BSs at the tagged femto user is obtained as follows

$$
\begin{aligned}
& \mathcal{L}_{I_{M B}}(s)=\mathrm{E}_{I_{M B}}\left[\exp \left(-s I_{M B}\right)\right] \\
= & \mathrm{E}_{\Phi_{M}, G_{i}}\left[\exp \left(-s \sum_{i \in \Phi_{M}} P_{M} G_{i} L_{i}^{-\alpha}\right)\right] \\
= & \mathrm{E}_{\Phi_{M}}\left[\prod_{i \in \Phi_{M}} \mathrm{E}_{G_{i}}\left[\exp \left(-s P_{M} G_{i} L_{i}^{-\alpha}\right)\right]\right]
\end{aligned}
$$

where $L_{i}$ is the distance of the $i$ th interfering macro-BS from the tagged femto receiver captured by the point process $\Phi_{M}$. The interference channel gains $G_{i}$ are assumed to be mutually independent and have identical pdfs (Nakagami- $\left.m_{I, M}\right)$. Each of the active interfering macro-BSs transmits with the same power $P_{M}$. Again, using the definition of the Generating functional for the PPP, we can write

$$
\mathcal{L}_{I_{M B}}(s)=\exp \left\{-\mathrm{E}_{G}\left[\int_{0}^{\infty}\left(1-\exp \left(-s P_{M} G L^{-\alpha}\right)\right) \lambda_{I}(L) \mathrm{d} L\right]\right\} .
$$

The interference is encountered from all the macroBSs located in the area $\mathbb{R}^{d} \backslash b(0,0)$. It should be noted that not all the macro-BSs in $\mathbb{R}^{d}$ will contribute towards the aggregate interference, i.e., only those macro-BSs transmitting on the same RB as the tagged femto user qualify as potential interferers. The intensity of the HPPP $\Phi_{M}$ process can be therefore written as follows

$$
\lambda_{I}(L)=\lambda_{M}^{\prime} d L^{d-1} b_{d}
$$

where $L$ is the distance between an arbitrary macro-BS and the tagged femto receiver and $\lambda_{M}^{\prime}$ is the intensity of those macro-BSs transmitting on the same RB as the tagged femto user at a time.

Now, (17) is rewritten as

$$
\exp \left\{-\mathrm{E}_{G}\left[\int_{0}^{\infty}\left(1-\exp \left(-s P_{M} G L^{-\alpha}\right)\right) \lambda_{M}^{\prime} L^{d-1} b_{d} d L^{d-1} \mathrm{~d} L\right]\right\} .
$$

By changing the variables and following a few simple steps, $\mathcal{L}_{I_{M B}}(s)$ is obtained as follows [11]

$$
\mathcal{L}_{I_{M B}}(s)_{\mid s=\frac{m_{d, F} \theta r_{F} \alpha}{\Omega_{d, F} P_{F}}}=e^{-b_{d} \lambda_{M}^{\prime} \Gamma\left(1-\frac{d}{\alpha}\right)\left(\frac{P_{M} \frac{m_{d, F}}{\Omega_{d, F}}}{P_{F}}\right)^{\frac{d}{\alpha}} r_{F}^{d} E\left[(G)^{\frac{d}{d}}\right]}
$$

Same as the desired link, we consider the Nakagami power fading model for the macro interfering links as well (Nakagami fading links with equal parameters $m_{I, M}$ and $\left.\Omega_{I, M}\right)$. Below, $\mathrm{E}\left[(G)^{\frac{d}{\alpha}}\right]$ is obtained using the definition of expectation and the standard gamma function

$$
\mathrm{E}\left[(G)^{\frac{d}{\alpha}}\right]=\frac{1}{\Omega_{I, M}}\left[\left(\frac{\Omega_{I, M}}{m_{I, M}}\right)^{\frac{d}{\alpha}+m_{I, M}}\left(\frac{m_{I, M} m_{I, M}}{\Gamma\left(m_{I, M}\right)}\right)\right] \Gamma\left(\frac{d}{\alpha}+m_{I, M}\right) .
$$

\subsubsection{Closed-form femto outage probability expression}

Finally, from (1), (8), (13), and (19) and by replacing $s=\frac{m_{d, F}}{\Omega_{d, F}} \frac{\theta r_{F}{ }^{\alpha}}{P_{F}}$, and $z=r_{F}{ }^{\alpha}$, the closed-form expression for the outage probability of the tagged femto user is obtained as follows

$$
p_{O F}=1-\overbrace{\left[\sum_{k=0}^{m_{d, F}-1} \frac{(z)^{k}}{k !}(-1)^{k} \frac{d^{k}(\exp (l(z)))}{d z^{k}}\right]}^{P[\operatorname{SINR}>\theta]}
$$

where

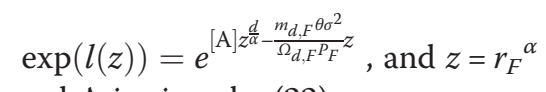

and $\mathrm{A}$ is given by (22).

$$
\mathrm{A}=\mathrm{A}_{1}+\mathrm{A}_{2}
$$

where

$$
\begin{gathered}
\mathrm{A}_{1}=b_{d} p_{R B} p_{t x} \lambda_{F}^{\prime}+\frac{d}{\alpha} b_{d} p_{R B} p_{t x} \lambda_{F}^{\prime}\left(\frac{\Gamma\left(-\frac{d}{\alpha}\right) m_{I, F}^{m_{I, F}}}{\Omega_{I, F}^{m_{I, F} \Gamma\left(m_{I, F}\right)}}\right) \\
\times\left[\sum_{k=0}^{\infty} \frac{\left(\frac{m_{d, F}}{\Omega_{d, F}}\right)^{k}\left(\frac{\Omega_{I, F} \Omega_{d, F}}{m_{I, F} \Omega_{d, F}+\Omega_{I, F} m_{d, F} \theta}\right)^{m_{I, F}+k} \Gamma\left(m_{I, F}+k\right)}{\Gamma\left(k+1-\frac{d}{\alpha}\right)}\right]
\end{gathered}
$$

and 


$$
\begin{aligned}
& \mathrm{A}_{2}=-b_{d} \lambda_{M}^{\prime} \Gamma\left(1-\frac{d}{\alpha}\right)\left(\frac{P_{M} \frac{m_{d, F}}{\Omega_{d, F}} \theta}{P_{F}}\right)^{\frac{d}{\alpha}} \\
& \times \frac{1}{\Omega_{I, M} m_{I, M}}\left[\left(\frac{\Omega_{I, M}}{m_{I, M}}\right)^{\frac{d}{\alpha}}+m_{I, M}\left(\frac{m_{I, M} m_{I, M}}{\Gamma\left(m_{I, M}\right)}\right)\right] \Gamma\left(\frac{d}{\alpha}+m_{I, M}\right) .
\end{aligned}
$$

Note: $m_{d, F}$ is constrained to take integer values only, while $m_{I, F}$ and $m_{I, M}$ can take any value (bigger than 0.5 ). The restriction on $m_{d, F}$ is because of $m_{d, F}-1$, as an upper limit for the summation in (21). However, for non-integer $m_{d, F}$ by using the infinite series representation of incomplete gamma function, we also obtain an exact but infinite summation expression for the outage probability of the tagged femto user as in Appendix 3.

The probability of outage averaged over the plane is derived as

$$
\begin{gathered}
p_{\bar{o} F}=1-\mathrm{E}_{r_{F}}[P[\operatorname{SINR}>\theta]] \\
p_{\bar{o} F}=1-\int_{0}^{\infty}[P[\operatorname{SINR}>\theta]] f_{r_{F}}\left(r_{F}\right) \mathrm{d} r_{F}
\end{gathered}
$$

where $f_{r_{F}}\left(r_{F}\right)=e^{-\lambda_{F} \pi r_{F}^{2}} 2 \pi \lambda_{F} r_{F}$ is the pdf of $r_{F}$ [6].

Here, for example, we present the $p_{\bar{o} F}$ in closed-form expressions for three different values of $m_{d, F}$ :

$$
\begin{aligned}
& m_{d, F}=1, \alpha=4: \quad p_{\bar{o} F}=1+\frac{\lambda_{F} \pi}{\left(\mathrm{A}-\lambda_{F} \pi\right)} \\
& m_{d, F}=2, \alpha=4: p_{\bar{o} F}=1+\frac{\lambda_{F} \pi\left[\left(\mathrm{A}-\lambda_{F} \pi\right)+\frac{\mathrm{A}}{2} \Gamma(2,0)\right]}{\left(\mathrm{A}-\lambda_{F} \pi\right)^{2}} \\
& m_{d, F}=3, \alpha=4: \\
& p_{\bar{o} F}=1+\frac{\lambda_{F} \pi\left[\left(\mathrm{A}-\lambda_{F} \pi\right)^{5}+\frac{5 \mathrm{~A}}{8} \Gamma(2,0)\left(\mathrm{A}-\lambda_{F} \pi\right)^{4}+\frac{\mathrm{A}^{2}}{8} \Gamma(3,0)\left(\mathrm{A}-\lambda_{F} \pi\right)^{3}\right]}{\left(\mathrm{A}-\lambda_{F} \pi\right)^{6}} .
\end{aligned}
$$

It should be noted that in all the above expressions $\left(\mathrm{A}-\lambda_{F} \pi\right)<0$, and $\sigma^{2} \rightarrow 0$ (operating in an interferencelimited regime). For $m_{d, F} \geq 4$, obtaining $p_{\bar{o} F}$ is also quite easy due to the fact that $\exp (l(z))$ is a factor common to every term of the all derivatives of $\exp (l(z))$.

\subsection{Macro outage probability}

We derive the outage probability for a typical macro user $\left(p_{O M}\right)$ defined as the probability that a randomly chosen macro user cannot achieve a target SINR $\gamma$, considering a collocated spectrum sensing CR-based femto network and macro-BSs as follows:

$$
p_{O M}=1-P[\operatorname{SINR}>\gamma]
$$

The experienced SINR by a typical macro user is calculated as

$$
\mathrm{SINR}=\frac{P_{M} h_{M} r_{M}^{-\alpha}}{\sigma^{2}+I_{M B}+I_{F B}}
$$

where $P_{M}$ is the transmission power from the nearest macro-BS located in the random distance $r_{M}$ from its tagged macro user, and $\alpha$ is the path-loss exponent. $I_{F B}$ and $I_{M B}$ are the aggregate interference power to the tagged macro user (located at the origin) from the surrounding femto-BSs and macro-BSs, respectively, and $\sigma^{2}$ is the noise power.

When the fading distribution is Nakagami- $m_{d, M}$ for the communication channel between the tagged macro user and its corresponding macro-BS, the pdf of the power fading coefficient $y=h_{M}$ is as follows

$$
p(y)=\frac{m_{d, M} m_{d, M}}{\Omega_{d, M} m_{d, M} \Gamma\left(m_{d, M}\right)}(y)^{m_{d, M}-1} e^{-\frac{m_{d, M} y}{\Omega_{d, M}}}
$$

where $m_{d, M}$ (a positive integer) is the fading parameter for the desired link, $\Omega_{d, M}$ is the average SNR. Next, the similar approach to obtain the outage probability of the tagged femto user is used for the outage probability calculation of the tagged macro user. Therefore, from (24) and (25) we have

$$
P[\operatorname{SINR}>\gamma]=\left[\sum_{k=0}^{m_{d, M}-1} \frac{(s)^{k}}{k !}(-1)^{k} \frac{d^{k}\left(\mathcal{L}_{I_{F B}}(s) \mathcal{L}_{I_{M B}}(s) e^{-s \sigma^{2}}\right)}{d s^{k}}\right]
$$

where $\mathcal{L}_{I_{F B}}(s)$ and $\mathcal{L}_{I_{M B}}(s)$ are the Laplace transform of random variables $I_{F B}$ and $I_{M B}$ evaluated at $s\left(s=\frac{m_{d, M}}{\Omega_{d, M}} \frac{\gamma r_{M}{ }^{\alpha}}{P_{M}}\right)$, respectively. We now calculate the closed-form expressions for $\mathcal{L}_{I_{F B}}(s)$ and $\mathcal{L}_{I_{M B}}(s)$ (at $s=\frac{m_{d, M}}{\Omega_{d, M}} \frac{\gamma r_{M}{ }^{\alpha}}{P_{M}}$ ).

\subsubsection{Computation of $\mathcal{L}_{I_{M B}}(s)$ and $\mathcal{L}_{I_{F B}}(s)$ at the tagged macro user}

Taking a similar approach to what we had before, $\mathcal{L}_{I_{M B}}$ $(s)$ is obtained as follows

$$
\mathcal{L}_{I_{M B}}(s)=\exp \left\{-\mathrm{E}_{g_{p}}\left[\int_{r_{M}}^{\infty}\left(1-\exp \left(-s P_{M} g_{p} W^{-\alpha}\right)\right) \lambda_{I}(W) \mathrm{d} W\right]\right\}
$$

and

$$
\lambda_{I}(W)=\lambda_{M}^{\prime} d W^{d-1} b_{d}
$$

in which $W$ is the distance between an arbitrary macroBS (captured by the point process $\Phi_{M}$ ) and the tagged macro receiver, and $\lambda_{M}^{\prime}$ is the intensity of those macroBSs transmitting on the same RB as the tagged macro 
user at a time. Similarly, the interference channel gains $g_{p}$ (between the interfering macro-BSs and the tagged macro user) are assumed to be mutually independent and have identical pdfs (Nakagami- $\left.m_{I, M}\right)$. Each of the active interfering macro-BSs transmits with the same power $P_{M}$ as for the tagged macro-BS. It should be noted that only those macro-BSs operating on the same $\mathrm{RB}$ as the tagged macro user and located in the area $\mathbb{R}^{d} \backslash b\left(0, r_{M}\right)$ make interference to the tagged macro user.

Same as before, (27) is simplified as

$$
\mathcal{L}_{I_{M B}}(s)_{\mid s=\frac{m_{d, M} \gamma r_{M} \alpha}{\Omega_{d, M} P_{M}}}=e^{\left.r_{M}{ }^{d} b_{d} \lambda_{M}^{\prime}-\frac{d}{\alpha} b_{d} \lambda_{M}^{\prime}\left(\frac{m_{d, M}}{\Omega_{d, M}}\right)\right)^{\frac{d}{\alpha}} r_{M}^{d} V(\gamma, \alpha)}
$$

in which $V(\gamma, \alpha)=\mathrm{E}\left[\left(g_{p}\right)^{\frac{d}{\alpha}}\left(\Gamma\left(-\frac{d}{\alpha}, \frac{m_{d, M}}{\Omega_{d, M}} \gamma g_{p}\right)-\Gamma\left(-\frac{d}{\alpha}\right)\right)\right]$.

The Nakagami power fading model is also considered for the interference links (Nakagami fading links with equal parameters $m_{I, M}$ and $\left.\Omega_{I, M}\right)$. The derivation of $V(\gamma$, $\alpha)$ is similar to the derivation of $M(\theta, \alpha)$ in Appendix 2 . Therefore, $V(\gamma, \alpha)$ is expressed as follows

$$
\begin{aligned}
& V(\gamma, \alpha)=\frac{-\Gamma\left(-\frac{d}{\alpha}\right) m_{I, M} m_{I, M}\left(\frac{m_{d, M}}{\Omega_{d, M}} \gamma\right)^{-\frac{d}{\alpha}}}{\Omega_{I, M} m_{I, M} \Gamma\left(m_{I, M}\right)} \\
& \times\left[\sum_{k=0}^{\infty} \frac{\left(\frac{m_{d, M}}{\Omega_{d, M}} \gamma\right)^{k}\left(\frac{\Omega_{I, M} \Omega_{d, M}}{m_{I, M} \Omega_{d, M}+\Omega_{I, M} m_{d, M} \gamma}\right)^{m_{I, M}+k}}{\Gamma\left(k+1-\frac{d}{\alpha}\right)} \Gamma\left(m_{I, M}+k\right)\right]
\end{aligned}
$$

Based on the same approach as described before, $\mathcal{L}_{I_{F B}}$ $(s)$ is also given by

$$
\mathcal{L}_{I_{F B}}(s)=\exp \left\{-\mathrm{E}_{G_{p}}\left[\int_{K_{r_{M}}}^{\infty}\left(1-\exp \left(-s P_{F} G_{p} U^{-\alpha}\right)\right) \lambda_{I}(U) \mathrm{d} U\right]\right\} .
$$

Since the closest interfering femto-BS is at least at distance $\mathrm{K}_{M}$ from the tagged macro user, the integration limits are from $\mathrm{K} r_{M}$ to $\infty$. In other words, interference is encountered from all the femto-BSs located in the area $\mathbb{R}^{d} \backslash b\left(0, K r_{M}\right)$ (see Figure 3). It should be noted that not all the femto-BSs outside this ball will contribute towards the aggregate interference, i.e., only those femto-BSs which are outside the mentioned ball and at minimum satisfy all of the following conditions are considered as potential contributors

(c) Satisfying the above condition, any arbitrary femtoBS contributes towards the interference at the tagged macro receiver, if it wrongly picks the same
$\mathrm{RB}$ as the tagged macro-BS to communicate with its user (we show the probability of picking a same RB for data transmission from a pool of all RBs as $p_{R B}$ (the calculation of $p_{R B}$ for this case is seen in Section 4, Scenario II, Case 2)).

(d)Same as the condition (b) in subsection 3.1.1.

Applying these two independent thinning, the intensity of the process (the number of the interfering CR femtoBSs at the tagged macro user) becomes

$$
\lambda_{I}(U)=\lambda_{F}^{\prime} d U^{d-1} b_{d} p_{R B} p_{t x} .
$$

Taking the similar approach, (31) is simplified as follows

$$
\mathcal{L}_{I_{F B}}(s)=e^{\mathrm{K}^{d} r_{M}{ }^{d} b_{d} p_{R B} p_{t x} \lambda_{F}^{\prime}-\frac{d}{\alpha} b_{d} p_{R B} p_{t x} \lambda_{F}^{\prime}\left(\frac{m_{d, M} P_{F} Y}{\Omega_{d, M} P_{M}}\right)^{\frac{d}{\alpha}} r_{M}{ }^{d} T(\gamma, \alpha)}
$$

in which $T(\gamma, \alpha)=\mathrm{E}\left[\left(G_{p}\right)^{\frac{d}{\alpha}}\left(\Gamma\left(-\frac{d}{\alpha}, \frac{\frac{m_{d, M}}{\Omega_{d, M}} \gamma P_{F} G_{p}}{K^{\alpha} P_{M}}\right)-\Gamma\left(-\frac{d}{\alpha}\right)\right)\right]$.

The Nakagami power fading model is also applied to the interference links (Nakagami fading links with equal parameters $m_{I, F}$ and $\left.\Omega_{I, F}\right)$. The mathematical derivation of $T(\gamma, \alpha)$ is quite similar to the derivation of $M(\theta, \alpha)$ and $V(\gamma, \alpha)$ (see Appendix 2). Hence, $T(\gamma, \alpha)$ is expressed as follows

$$
\begin{aligned}
& T(\gamma, \alpha)=\frac{-\Gamma\left(-\frac{d}{\alpha}\right) m_{l, F} m_{I, F}\left(\frac{m_{d, M}}{\Omega_{d, M} \mathrm{~K}^{\alpha} P_{M}} \gamma\right)^{-\frac{d}{\alpha}}}{\Omega_{I, F}^{m_{I, F}} \Gamma\left(m_{l, F}\right)} \\
& \times\left[\sum_{k=0}^{\infty} \frac{\left(\frac{\frac{m_{d, M}}{\Omega_{d, M}} \gamma P_{F}}{\mathrm{~K}^{\alpha} P_{M}}\right)^{k}\left(\frac{\mathrm{K}^{\alpha} P_{M} \Omega_{d, M} \Omega_{I, F}}{m_{I, F} \Omega_{d, M} \mathrm{~K}^{\alpha} P_{M}+m_{d, M} \Omega_{I, F} P_{F} \gamma}\right)^{m_{l, F}+k}}{\Gamma\left(k+1-\frac{d}{\alpha}\right)} \Gamma\left(m_{I, F}+k\right)\right] .
\end{aligned}
$$

\subsubsection{Closed-form macro outage probability expression}

Finally, from (24), (26), (29), and (33) and by replacing $s=\frac{m_{d, M}, \frac{\gamma r_{M}{ }^{\alpha}}{\Omega_{d, M}}}{P_{M}}$, and $z=r_{M}{ }^{\alpha}$, the closed-form expression for the outage probability of the tagged macro user is obtained as follows

$$
p_{O M}=1-\left[\sum_{k=0}^{m_{d, M}-1} \frac{(z)^{k}}{k !}(-1)^{k} \frac{d^{k}(\exp (l(z)))}{d z^{k}}\right]
$$

where

$$
\exp (l(z))=e^{[\mathrm{B}] z^{\frac{d}{\alpha}-}-\frac{m_{d, M}, \sigma^{2}}{\Omega_{d, M} P_{M}} z} \text { and } z=r_{M}{ }^{\alpha}
$$

and $\mathrm{B}$ is given by (36). 


$$
\mathrm{B}=\mathrm{B}_{1}+\mathrm{B}_{2}
$$

where

$$
\begin{gathered}
\mathrm{B}_{1}=\mathrm{K}^{d} b_{d} p_{R B} p_{t x} \lambda_{F}^{\prime}+\frac{d}{\alpha} b_{d} p_{R B} p_{t x} \lambda_{F}^{\prime}\left(\frac{m_{d, M}}{\Omega_{d, M}} \frac{\gamma}{P_{M}}\right)^{\frac{d}{\alpha}} \\
\times\left(\frac{\Gamma\left(-\frac{d}{\alpha}\right) m_{I, F} m_{I, F}\left(\frac{m_{d, M}}{\Omega_{d, M} \mathrm{~K}^{\alpha} P_{M}} \gamma\right)^{-\frac{d}{\alpha}}}{\Omega_{I, F} m_{l, F} \Gamma\left(m_{I, F}\right)}\right) \\
\times\left[\sum_{k=0}^{\infty} \frac{\left(\frac{\frac{m_{d, M}}{\Omega_{d, M}} \gamma P_{F}}{\mathrm{~K}^{\alpha} P_{M}}\right)^{k}\left(\frac{\mathrm{K}^{\alpha} P_{M} \Omega_{d, M} \Omega_{I, F}}{m_{I, F} \Omega_{d, M} \mathrm{~K}^{\alpha} P_{M}+m_{d, M} \Omega_{I, F} P_{F} \gamma}\right)^{m_{I, F}+k}}{\Gamma\left(k+1-\frac{d}{\alpha}\right)} \Gamma\left(m_{I, F}+k\right)\right] .
\end{gathered}
$$

and

$$
\begin{gathered}
\mathrm{B}_{2}=b_{d} \lambda_{M}^{\prime}+\frac{d}{\alpha} b_{d} \lambda_{M}^{\prime}\left(\frac{\Gamma\left(-\frac{d}{\alpha}\right) m_{I, M} m_{I, M}}{\Omega_{I, M} m_{I, M} \Gamma\left(m_{I, M}\right)}\right) \\
\times\left[\sum_{k=0}^{\infty} \frac{\left(\frac{m_{d, M}}{\Omega_{d, M}} \gamma\right)^{k}\left(\frac{\Omega_{I, M} \Omega_{d, M}}{m_{I, M} \Omega_{d, M}+\Omega_{I, M} m_{d, M} \gamma}\right)^{m_{I, M}+k}}{\Gamma\left(k+1-\frac{d}{\alpha}\right)} \Gamma\left(m_{I, M}+k\right)\right] .
\end{gathered}
$$

The probability of outage averaged over the plane can also be derived as

$$
\begin{gathered}
p_{\bar{o} M}=1-\mathrm{E}_{r_{M}}[P[\text { SINR }>\gamma]] \\
p_{\bar{o} M}=1-\int_{0}^{\infty}[P[\text { SINR }>\gamma]] f_{r_{M}}\left(r_{M}\right) \mathrm{d} r_{M}
\end{gathered}
$$

where $f_{r_{M}}\left(r_{M}\right)=e^{-\lambda_{M} \pi r_{M}{ }^{2}} 2 \pi \lambda_{M} r_{M}$ is the pdf of $r_{M}$. Such as before, $p_{\bar{o} M}$ can be easily obtained for $m_{d, M}=1$, $2,3, \ldots$.

\subsection{Outage probability formulation under perfect and imperfect sensing}

We now study the effect of both the ideal detection, i.e., $P_{d}=1$ and $P_{f}=0$, and the cases involving imperfect sensing, i.e., $P_{d} \neq 1$ and $P_{f} \neq 0$ on the outage probabilities of femto and macro users.

\subsubsection{Scenario I}

Ideal detection $\left(P_{d}=1\right.$ and $\left.P_{f}=0\right)$ :

3.3.1.1 (femto and macro outage probabilities) Each secondary node (femto-BS) has perfect knowledge of each primary (macro-BS) signaling. In other words, sensing at each femto-BS is done perfectly. Therefore, RBs occupied by the macro network are not chosen by any femo-BS for data transmission. In this case, the tagged femto user does not experience any interference from the macro-BSs. This assumption $\left(P_{d}=1\right.$ and $\left.P_{f}=0\right)$ is reasonable in the scenarios where interference from other systems is assumed to be negligible.

Therefore, under this assumption, $\mathrm{E}\left[e^{-s I_{M B}}\right]=1$ and $\mathcal{L}_{I_{M B}}(s)=1$ (in (7)), consequently. Then, for the outage probability of the tagged femto user in (21), A is only equal to $A_{1}$ (see (22)). It should be noted that the calculation of $p_{R B}$ for this case is presented in Section 4, Scenario I, Case 1.

Clearly, under this scenario and considering the above explanations, the tagged macro user does not experience any interference from the surrounding femto-BSs. Therefore, for the outage probability of the tagged macro user in (35), $B$ is only equal to $B_{2}$ (see (36)).

\subsubsection{Scenario II}

Imperfect detection $\left(P_{d} \neq 1\right.$ and $\left.P_{f} \neq 0\right)$ :

3.3.2.1 (femto and macro outage probabilities) In this scenario, each secondary node (femto-BS) has imperfect knowledge of each primary (macro-BS) signaling. In other words, sensing at each femto-BS is done imperfectly and subject to (occasional) error. Therefore, RBs occupied by the macro network may be wrongly chosen by the femo-BSs, as well. Two cases can take place under the imperfect sensing scenario:

Case 1. The tagged femto-BS transmits data on an idle $\mathrm{RB}$ (in this case, the outage probability formulations (for both the tagged femto and macro users) are the same as in the perfect sensing scenario except for the calculation of $p_{R B}$ (see Section 4, Scenario II, Case 1))

Case 2. The tagged femto-BS transmits on an occupied $\mathrm{RB}$ (in this case, the outage probability formulations for both the tagged femto and macro users are explained as follows and the calculation of $p_{R B}$ is presented in Section 4, Scenario II, Case 2)

In Case 2, the tagged femto user can experience interference from both the active femto-BSs (which pick the same occupied RB as the tagged femto-BS) and macroBSs. Then, for the outage probability of the tagged femto user in (21), $\mathrm{A}$ is equal to $\mathrm{A}_{1}+\mathrm{A}_{2}$ (see (22)). Clearly, under this scenario and in Case 2, the tagged macro user can experience interference on its $\mathrm{RB}$, from both the femto-BSs and macro-BSs. Indeed, for the outage probability of the tagged macro user in (35), $\mathrm{B}$ is equal to $\mathrm{B}_{1}+\mathrm{B}_{2}$.

\section{Resource block selection probability $\left(p_{R B}\right)$ calculations under perfect and imperfect sensing}

In this section, we discuss how the optimal values of the RB selection probability $\left(p_{R B}\right)$ for a secondary transmitter (femto-BS) can be determined under each femto-BS's perfect and imperfect sensing scenarios. 


\subsection{Scenario I}

Ideal detection for all the CR femto-BSs $\left(P_{d}=1\right.$ and $P_{f}=$ 0) $[13,14]$ :

Case 1. The tagged femto-BS assigns the $i$ th idle RB to its femto user.

$p_{R B}$ : The probability that the $i$ th idle RB being selected for data transmission by any of the other active $C R$ femto-BS [14].

$$
p_{R B \mid M_{s}}=p_{\text {idle }}\left(M_{s}\right) \cdot \frac{\left(\begin{array}{c}
M-1 \\
M_{s}-1
\end{array}\right)}{\left(\begin{array}{c}
M \\
M_{s}
\end{array}\right)} \cdot\left(\frac{1}{M_{s}}\right)
$$

where

$$
p_{\text {idle }}\left(M_{s}\right)=\frac{\left(\begin{array}{c}
M \\
M_{s}
\end{array}\right)\left(\begin{array}{c}
N-M \\
N_{s}-M_{s}
\end{array}\right)}{\left(\begin{array}{c}
N \\
N_{s}
\end{array}\right)} .
$$

The first term $\left(p_{\text {idle }}\left(M_{s}\right)\right)$ indicates the probability of $M_{s}$ idle RBs sensed by one femto-BS (during the sensing time, $T_{S}$ ) and the second term is the probability that the $i$ th idle RB is inside the $M_{s}$ idle RBs, and finally the third term indicates that the probability of selecting the $i$ th idle RB (out of the $M_{s}$ idle RBs) by that femto-BS is equal to $\frac{1}{M_{s}}$ (since each of the idle RBs within the $M_{s}$ idle RBs has an equal probability of being chosen). It should be noted that each CR femto-BS will fail to access when $M_{s}=0$ (the maximum value of $M_{s}$ is equal to $\min \{M$, $\left.N_{s}\right\}$ ). Therefore, from (38),

$$
\begin{aligned}
p_{R B} & =p_{R B \mid\left(M_{s} \geq 1\right)}=\frac{1}{M} p_{\text {idle }}\left(M_{s} \geq 1\right) \\
& =\frac{1}{M}\left(1-p_{\text {idle }}\left(M_{s}=0\right)\right) .
\end{aligned}
$$

If the number of RBs sensed by one CR femto-BS (i.e., $N_{s}$ ) is more than or equal to $N-M+1$, then at least we have one idle RB within the $N_{s}$ detected RBs, i.e., $p_{\text {idle }}\left(M_{s}=0\right)=0$. On the other hand, if $N_{s}$ is smaller than or equal to $N-M$, the CR femto-BS will fail to access when the RBs within the $N_{s}$ sensed RBs are all occupied by the macro-BSs. Therefore, we can write

$$
p_{\text {idle }}\left(M_{s}=0\right)=\left\{\begin{array}{cc}
\frac{\left(\begin{array}{c}
N-M \\
N_{s}
\end{array}\right)}{\left(\begin{array}{c}
N \\
N_{s}
\end{array}\right),} & \text { if } N_{s} \leq N-M \\
0, & \text { if } N_{s} \geq N-M+1 .
\end{array}\right.
$$

From (39) and (40), the probability that a CR femto-BS selects the $i$ th idle RB for data transmission is obtained as follows

$$
p_{R B}=\left\{\begin{array}{cc}
\frac{1}{M}\left(\begin{array}{c}
\left.1-\frac{\left(\begin{array}{c}
N-M \\
N_{s}
\end{array}\right)}{\left(\begin{array}{c}
N \\
N_{s}
\end{array}\right)}\right), \\
\frac{1}{M},
\end{array} \quad \text { if } N_{s} \leq N-M\right. \\
\text { if } N_{s} \geq N-M+1 .
\end{array}\right.
$$

\subsection{Scenario II}

Imperfect detection for all the CR femto-BSs $\left(P_{d} \neq 1\right.$ and $\left.P_{f} \neq 0\right)[13,14]:$

Case 1. The tagged femto-BS assigns the $i$ th idle RB to its femto user.

$p_{R B}$ : The probability that the $i$ th idle RB being selected for data transmission by any of the other active $C R$ femto-BS [14].

We show the detection result indicator of the $n$th RBs by $D_{n}(n \in\{1,2, \ldots, N\})$. If $D_{n}=1$, the $n$th RB is detected as idle RB; otherwise, $D_{n}=0$. The probability of one idle $\mathrm{RB}$ detected with no false alarm is $1-P_{f}$ and the probability for an occupied RB detected as an idle RB is 1 $P_{d}$. In other words,

$$
\operatorname{Pr}\left(D_{n}=1\right)= \begin{cases}V_{0}=1-P_{f}, & \text { if } n \text {th RB isidle } \\ V_{1}=1-P_{d}, & \text { if } n \text {th RB is busy }\end{cases}
$$

in which $P_{f}$ is the false alarm probability and can be obtained as follows [14-16]

$$
\begin{gathered}
P_{f}(\tau)=\mathcal{Q}\left(\sqrt{2 \eta+1} \mathcal{Q}^{-1}\left(P_{d}\right)+\sqrt{\tau f_{s}} \eta\right) \\
\left(\mathcal{Q}(x)=\frac{1}{\sqrt{2 \pi}} \int_{x}^{\infty} \exp \left(\frac{-t^{2}}{2}\right) \mathrm{d} t \text { and } P_{d}\right. \text { is the predefined }
\end{gathered}
$$

detection probability. $\tau$ is the spectrum sensing time, $f_{s}$ the sampling frequency, and $\eta$ the received interference power on an RB to each femto-BS).

Indeed, the probability that the $i$ th idle $\mathrm{RB}$ is detected with no false alarm by a CR femto-BS is $\operatorname{Pr}\left(D_{i}=1\right)=V_{0}$.

To obtain the probability that the $i$ th idle RB being selected for data transmission by one CR femto-BS $\left(p_{R B}\right)$, first, we calculate the probability that the $i$ th idle $\mathrm{RB}$ is sensed and included in the $M_{s}$ idle RBs out of the $N_{s}$ sensed RBs in the sensing period $\left(T_{S}\right)$, and it is expressed as follows

$$
\begin{gathered}
\operatorname{Pr}\left(\text { the } i \text { th idle RB is sensed } \mid M_{s}\right) \\
=p_{\text {idle }}\left(M_{s}\right) \cdot \frac{\left(\begin{array}{c}
M-1 \\
M_{s}-1
\end{array}\right)}{\left(\begin{array}{c}
M \\
M_{s}
\end{array}\right)}
\end{gathered}
$$

where 


$$
p_{\text {idle }}\left(M_{s}\right)=\frac{\left(\begin{array}{c}
M \\
M_{s}
\end{array}\right)\left(\begin{array}{c}
N-M \\
N_{s}-M_{s}
\end{array}\right)}{\left(\begin{array}{c}
N \\
N_{s}
\end{array}\right)}
$$

$$
p_{R B}=\sum_{M_{s}=\max \left\{1, N_{s}-(N-M)\right\}}^{\min \left\{N_{s}, M\right\}} \sum_{M_{D}=1}^{N_{s}} p_{R B \mid M_{s}, M_{D}}
$$

Conditioning on $M_{D}$ (see Table 1) and $M_{s}$, the probability that the $i$ th idle RB being detected as idle is obtained as follows

$$
\begin{gathered}
\operatorname{Pr}\left(D_{i}=1 \mid M_{s}, M_{D}\right) \\
=\operatorname{Pr}\left(D_{i}=1\right) \cdot \operatorname{Pr}\left(\sum_{n \neq i, n \in \Phi} D_{n}=M_{D}-1 \mid M_{s}\right) \\
\sum_{0} \times\left[\begin{array}{c}
\min \left\{M_{D}, M_{s}\right\} \\
m_{I D}=\max \left\{1, M_{D}-\left(N_{s}-M_{s}\right)\right\} \\
\cdot\left(\begin{array}{c}
M_{s}-1 \\
N_{S}-M_{s} \\
m_{O D}-1
\end{array}\right) \cdot\left(V_{0}\right)^{m_{I D}-1}\left(1-V_{0}\right)^{M_{s}-m_{I D}}\left(1-V_{1}\right)^{\left.N_{s}-M_{s}-m_{O D}\right]}
\end{array}\right]
\end{gathered}
$$

in which $\Phi$ is the set of the detected RBs by one femtoBS (see Table 1 for the definitions of $m_{I D}$ and $m_{O D}$ ). Replacing $m_{O D}$ with $M_{D}-m_{I D}$, we have

$$
\begin{aligned}
\operatorname{Pr}\left(D_{i}\right. & \left.=1 \mid M_{s}, M_{D}\right) \\
& =\left[\begin{array}{c}
\sum_{\substack{\min \left\{M_{D}, M_{s}\right\} \\
m_{I D}=\max \left\{1, M_{D}-\left(N_{s}-M_{s}\right)\right\}}}^{\left(\begin{array}{c}
N_{s}-M_{s} \\
M_{D}-m_{I D}
\end{array}\right) \cdot\left(V_{1}\right)^{M_{D}-m_{I D}}\left(1-V_{1}\right)^{\left.N_{s}-M_{s}-M_{D}+m_{I D}\right]}}\left[\begin{array}{c}
M_{s}-1 \\
m_{I D}-1
\end{array}\right)\left(V_{0}\right)^{m_{I D}}\left(1-V_{0}\right)^{M_{s}-m_{I D}}
\end{array}\right]
\end{aligned}
$$

Having the $M_{D}$ detected idle RBs (including the $i$ th idle $\mathrm{RB}$ ), the probability of a CR femto-BS accessing the $i$ th idle RB is equal to $\frac{1}{M_{D}}$. Thus, the probability that the $i$ th idle RB is selected for data transmission by any $\mathrm{CR}$ femto-BS (under imperfect sensing scenario) is obtained as follows

$$
\begin{aligned}
p_{R B \mid M_{s}, M_{D}}= & \operatorname{Pr}\left(\text { the } i \text { th idle } \mathrm{RB} \text { is sensed } \mid M_{s}\right) \\
& \times \frac{1}{M_{D}} \times \operatorname{Pr}\left(D_{i}=1 \mid M_{s}, M_{D}\right) .
\end{aligned}
$$

Finally,

\section{Table 1 Symbols used in Section 4}

\begin{tabular}{ll}
\hline Symbol & Definition \\
\hline$N$ & Number of RBs \\
$M$ & Number of idle RBs \\
$N_{s}$ & Number of sensed RBs \\
$M_{s}$ & Number of idle RBs within the $N_{s}$ sensed RBs \\
$M_{D}$ & Number of RBs detected as idle within the $N_{s}$ sensed RBs \\
$m_{I D}$ & Number of idle RBs (out of the $M_{D}$ detected idle RBs) detected \\
& correctly. ( $m_{I D} \in\left[\right.$ max $\left\{1, M_{s}-\left(N_{s}-M_{D}\right)\right\}$, min $\left.\left\{M_{s}, M_{D}\right\}\right]$ ) \\
$m_{O D}$ & $\begin{array}{l}\text { Number of busy RBs (out of the } M_{D} \text { detected idle RBs) } \\
\left.\text { detected as idle. ( } m_{O D}=M_{D}-m_{I D}\right)\end{array}$ \\
\hline
\end{tabular}

Case 2. The tagged femto-BS assigns the $i$ th busy RB (occupied by the macro network) to its femto user.

$p_{R B}$ : The probability that the $i$ th busy RB being selected for data transmission by any of the other active CR femto-BS.

To obtain the probability of the $i$ th busy RB selected for data transmission by one femto-BS $\left(p_{R B}\right)$, first, we calculate the probability that the $i$ th busy RB is sensed and included in the $\left(N_{s}-M_{s}\right)$ busy RBs out of the $N_{s}$ sensed RBs in the sensing period, and it is expressed as follows

Conditioning on $M_{D}$ and $\left(N_{s}-M_{s}\right)$, the probability that the $i$ th busy RB being detected as idle is obtained as follows

$$
\begin{aligned}
& \begin{array}{c}
\operatorname{Pr}\left(D_{i}=1 \mid\left(N_{s}-M_{s}\right), M_{D}\right) \\
=\operatorname{Pr}\left(D_{i}=1\right) \cdot \operatorname{Pr}\left(\sum_{n \neq i, n \in \Phi} D_{n}=M_{D}-1 \mid\left(N_{s}-M_{s}\right)\right)
\end{array} \\
& =V_{1} \times\left[\begin{array}{c}
\sum_{m_{I D}=\max \left\{1, M_{D}-\left(N_{s}-M_{s}\right)\right\}}^{\min \left\{M_{D}, M_{s}\right\}}\left[\left(\begin{array}{c}
M_{s} \\
m_{I D}
\end{array}\right)\left(V_{0}\right)^{m_{I D}}\left(1-V_{0}\right)^{M_{s}-m_{I D}}\right. \\
\cdot\left(\begin{array}{c}
N_{s}-M_{s}-1 \\
m_{O D}-1
\end{array}\right) \cdot\left(V_{1}\right)^{m_{O D}-1}\left(1-V_{1}\right)^{\left.N_{s}-M_{s}-m_{O D}\right]}
\end{array}\right]
\end{aligned}
$$

in which $\Phi$ is the set of the detected RBs by a femto-BS. Again, replacing $m_{O D}$ with $M_{D}-m_{I D}$, we have

$$
=\left[\begin{array}{c}
\operatorname{Pr}\left(D_{i}=1 \mid\left(N_{s}-M_{s}\right), M_{D}\right) \\
\sum_{\min \left\{M_{D}, M_{s}\right\}}^{m_{I D}=\max \left\{1, M_{D}-\left(N_{s}-M_{s}\right)\right\}}\left[\left(\begin{array}{c}
M_{s} \\
m_{I D}
\end{array}\right)\left(V_{0}\right)^{m_{I}}\left(1-V_{0}\right)^{M_{s}-m_{I D}}\right. \\
\left.\cdot \begin{array}{c}
N_{s}-M_{s}-1 \\
M_{D}-m_{I D}-1
\end{array}\right) \cdot\left(V_{1}\right)^{M_{D}-m_{I D}}\left(1-V_{1}\right)^{\left.N_{s}-M_{s}-M_{D}+m_{I D}\right]}
\end{array}\right]
$$

Having the $M_{D}$ detected idle RBs (including the $i$ th busy $\mathrm{RB}$ ), the probability of a CR femto-BS accessing the $i$ th busy RB is $\frac{1}{M_{D}}$. Thus, the probability that the $i$ th busy $\mathrm{RB}$ is selected for data transmission by any CR femto-BS (under imperfect sensing scenario) is obtained as follows

$$
\begin{aligned}
p_{R B \mid\left(N_{s}-M_{s}\right), M_{D}} & =\operatorname{Pr}\left(\text { the } i \text { th idle RBis sensed } \mid\left(N_{s}-M_{s}\right)\right) \times \frac{1}{M_{D}} \\
& \times \operatorname{Pr}\left(D_{i}=1 \mid\left(N_{s}-M_{s}, M_{D}\right)\right) .
\end{aligned}
$$

Finally,

$$
\operatorname{Pr}\left(\text { the } i \text { th busy RB is sensed } \mid\left(N_{s}-M_{s}\right)\right)=\frac{\left(\begin{array}{c}
N-M-1 \\
N_{s}-M_{s}-1
\end{array}\right)\left(\begin{array}{c}
M \\
M_{s}
\end{array}\right)}{\left(\begin{array}{c}
N \\
N_{s}
\end{array}\right)} .
$$




$$
p_{R B}=\sum_{M_{s}=\max \left\{1, N_{s}-(N-M)\right\}}^{\min \left\{N_{s}, M\right\}} \sum_{M_{D}=1}^{N_{s}} p_{R B \mid\left(N_{s}-M_{s}\right), M_{D}} .
$$

\section{Simulation results and discussions}

Outage probability is considered as a QoS metric to meet a specific connection data rate requirement, or it can be utilized as a performance measure to evaluate the level of meeting the total demands of users in highly dense networks such as the heterogeneous cellular networks. By obtaining an exact closed-form expression for the system outage probability, we will be able to take a finer look at the system performance with the end goal of better understanding system design principles for the heterogeneous cellular networks.

Outage probability is affected by many parameters that have impact on the system performance. As mentioned before, cognition can be a potential solution for dynamic spectrum allocation which will adapt to the network geometry, solve the interference management issue, and reduce the outage probability. Therefore, the primary motivations for the femto-BSs to employ this cognition and hence, the $p_{R B}$ and $p_{t x}$ parameters in the cellular network are to provide beneficial coverage improvements for different types of users. In practical heterogeneous cellular networks such as LTE, these parameters are network-specific and not user-specific; thus, there needs to be some optimization techniques, which can be considered as future work, used to select parameters that can provide acceptable QoS for the majority of users and to meet the existing outage constraints.

On the other hand, outage probability is significantly affected by the performance of the spectrum sensing. The performance of the spectrum sensing and the determination of each individual RB as busy/idle is determined by two important parameters mentioned before, namely the probability of (correct) detection $\left(P_{d}\right)$ and probability of false alarm $\left(P_{f}\right)$. Considering the fact that $p_{R B}$ is a direct function of $P_{f}$ and $P_{d}$ in the practical scenarios (see Section 4), a spectrum sensing with high $P_{f}$ and low $P_{d}$ results in a lower corresponding QoS and a higher observable outage probability. The ideal sensing procedure is the one with $P_{f}=0$ and $P_{d}=1$. In practice, however, $P_{d}$ and $P_{f}$ are related to each other through a receiver operating characteristic (ROC) curve [17], which is a fundamental attribute of each spectrum sensing system.

We will see later that the outage probability is also an increasing function of the SINR threshold as theoretically can be proved. The SINR threshold $\theta$ is generally under the control of the system designer and should be chosen reasonably. Clearly, setting a high value for $\theta$ maintains the signal quality but increases the outage probability and reduces the network utilization. Therefore, an upper bound of this threshold can be considered [18] in order to guarantee that outage probability is kept below a maximum value. On the other hand, lowering $\theta$ is desirable for better resource utilization. However, small $\theta$ allows more users to be admitted per RB which can make the system infeasible if the number of admitted users per RB exceeds a certain limit.

First, the accuracy of our analytical results for the downlink analysis in the proposed model is validated by simulations, as shown in Figures 4 and 5. The simulations which are built on Matlab platform are carried out to plot the curves of outage probability versus the SINR threshold for the tagged femto and macro user, as shown in Figures 4 and 5, respectively. The considered scenario is a two-tier network (exactly following the network model described in Sections 2 and 3) over an approximately $1 \times 1 \mathrm{~km}$ square with the locations of different classes of BSs as realizations of independent PPPs of given densities, and the tagged users located at the center. To have an estimate of the outage probability at the tagged users, the simulation results are averaged over both the spatial PPP (500 different positions) and fading distribution (300 realizations), and are conducted using the parameters mentioned in the figures' captions. Analytical curves are compared with the simulations under both perfect and imperfect sensing. It is observed that the simulation results closely match our analytical model and the curves of analytical and simulation results match fairly well, which confirms our analysis. The plots exhibit slight discrepancies between analytical results and the corresponding simulation results which are mainly due

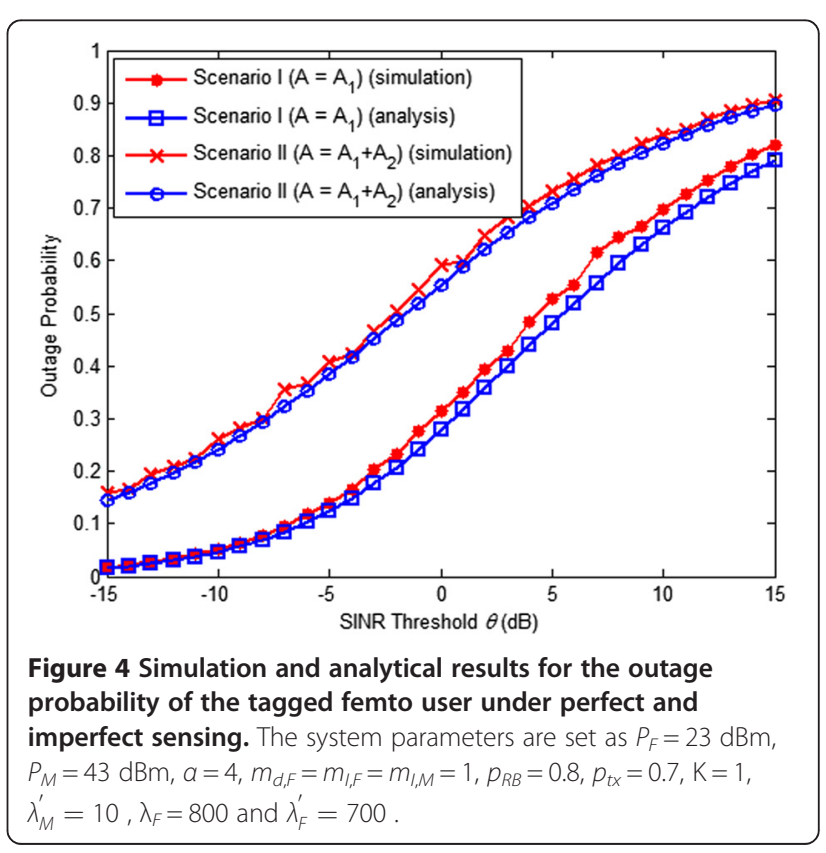




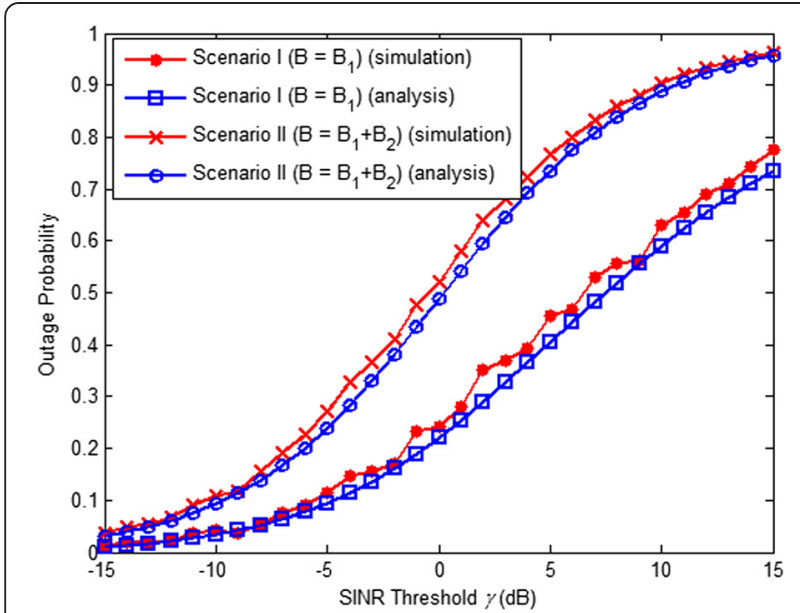

Figure 5 Simulation and analytical results for the outage probability of the tagged macro user under perfect and imperfect sensing. The system parameters are set as $P_{F}=23 \mathrm{dBm}$, $P_{M}=43 \mathrm{dBm}, a=4, m_{d, M}=m_{l, M}=m_{l, F}=1, p_{R B}=0.8, p_{t x}=0.7, \mathrm{~K}=1$, $\lambda_{F}^{\prime}=700, \lambda_{M}^{\prime}=10$ and $\lambda_{M}=50$.

to the boundary effect and the independence assumption used in Section 3.

In Figure 6, the outage probability of the tagged femto user under perfect and imperfect spectrum sensing abilities for the CR femto-BSs is shown for different values of the target SINR $\theta$ on the horizontal axis. Our results show that the outage probability at the tagged femto receiver in the absence of a perfect spectrum sensing ability is considerably increased. Either in Scenario I, when all the femto-BSs employ perfect sensing to sense the RBs, or in Scenario II, Case 1, the tagged femto user does not experience any interference from the macro-

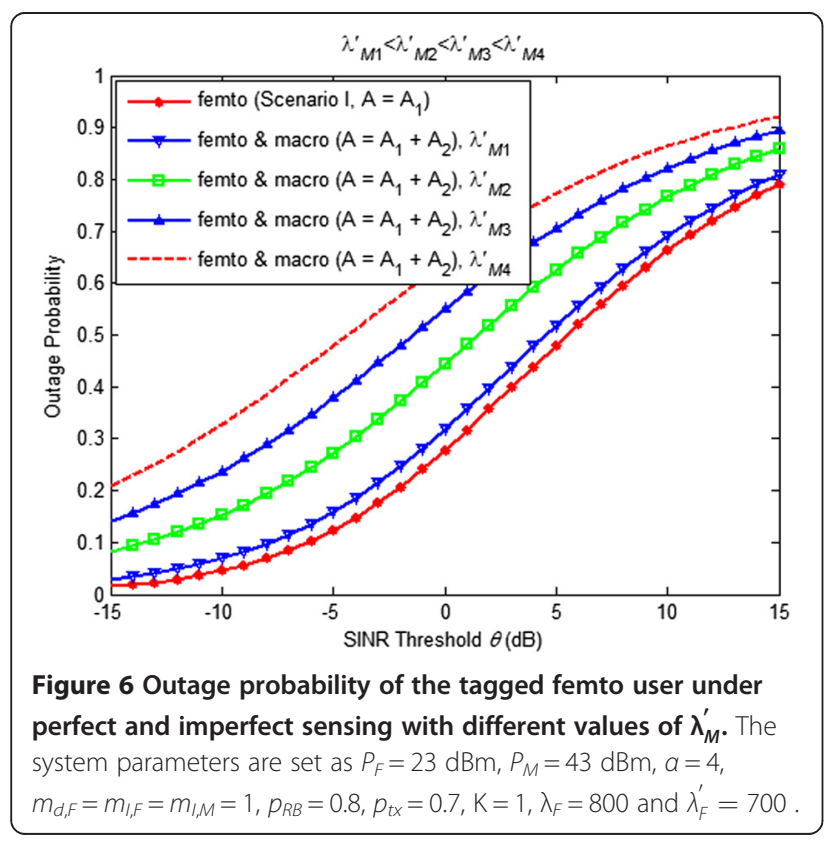

BSs owing to the correct detection at the tagged femtoBS or choice of idle RBs for data transmission (RBs not occupied by the macro network). In this case, the interference seen by the tagged femto user is only the aggregate interference from the other femto-BSs which are transmitting on the same idle RB as the tagged femto. Clearly, the lowest outage probability is for the case of perfect sensing $\left(A=A_{1}\right.$ in (22)). Now, let's consider the imperfect sensing scenario for the CR femto-BSs. Obviously, the tagged femto user is now subject to sensing error and therefore picking an occupied RB for its data transmission period. Under this condition, it may receive interference not only from the other femto-BSs which pick the same busy RB (due to the imperfect sensing), but also from those macro-BSs communicating with their own users on the same RB as the tagged femto. Therefore, the tagged femto user experiences an interference larger than before and consequently a significant increase in the outage probability. Moreover, the tagged femto user will face an outage with a higher probability whenever it picks an RB (occupied RB) already used by a larger number of macro users (a larger $\lambda_{M}^{\prime}$ ) (see Figure 6).

Figure 7 depicts the outage probability of the tagged macro user for different values of the target SINR $\gamma$ and different situations. Considering the results obtained in Figure 6, here, we also investigate the effect of employing the two different sensing scenarios for the $C R$ femto-BSs on the outage probability of the tagged macro user. In the case of perfect sensing, the tagged macro user does not experience any interference from the femto-BSs because only those RBs sensed to be idle (RBs not occupied by the macro network) are always chosen for data transmission by the femto network. In this case,

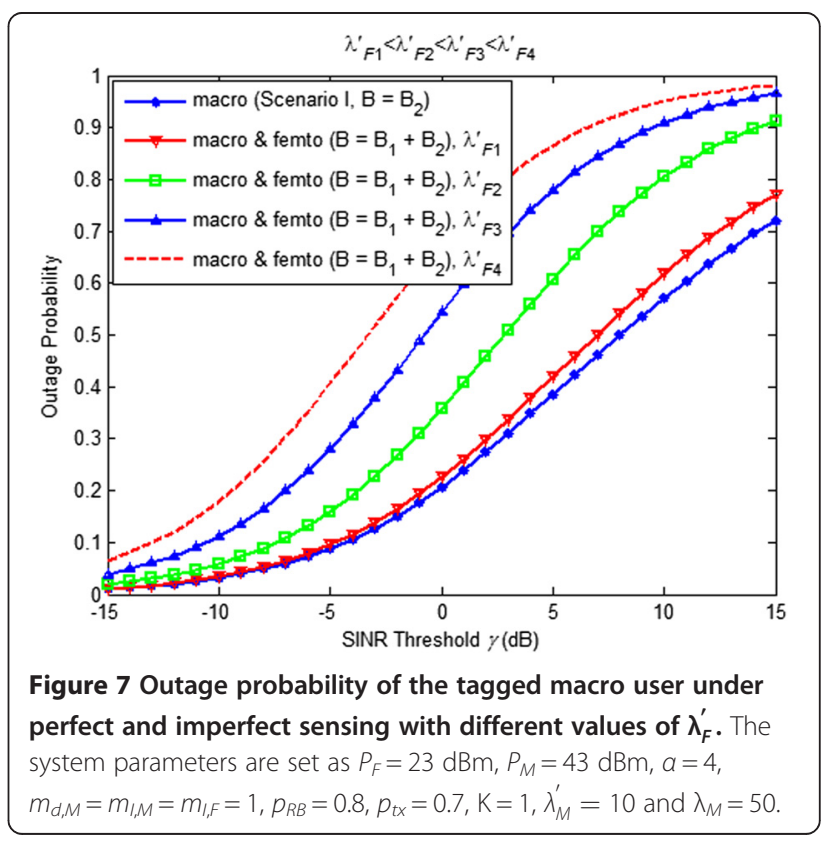


the interference observed at the tagged macro user is only the aggregate interference received from those macro-BSs transmitting on the same RB as the tagged macro. Clearly, the lowest outage probability is obtained for this case $\left(B=B_{2}\right.$ in (36)). Now, the case of imperfect spectrum sensing of the CR femto-BS nodes is considered, when the femto-BSs are subject to sensing error and therefore the possibility of transmitting on the RB occupied by the tagged macro. Under this condition, the tagged macro user may receive interference not only from the other macro-BSs communicating with their own users over the same RB (due to the lack of RBs) as the tagged macro, but also from those femto-BSs which pick the same RB. Therefore, the tagged macro user experiences an interference larger than before and consequently a significant increase in the outage probability. Moreover, the tagged macro user will face an outage with a higher probability whenever its own RB is wrongly selected for data transmission by a larger number of femto-BSs (a larger $\lambda_{F}^{\prime}$ ).

Figure 8 illustrates the effect of $\mathrm{K}$ (in $D=\mathrm{K} r_{M}$ ) on the observed outage probability at the tagged macro user in the presence of both the macro and femto networks. Considering the previous explanations and Figure 3, let $\Phi_{F}^{\prime}$ include all the points (representing the femto-BSs) in $\Phi_{F}$ except the points inside the exclusion region $D$ of the tagged macro user. Since $\Phi_{F}^{\prime} \subset \Phi_{F}$, the potential aggregate interference at the tagged macro user, caused by the active (considering slotted ALOHA) CR femto-BSs is less than that in the case with no $D$. Furthermore, as the exclusion region $D$ becomes larger (when $\mathrm{K}=10$, for example), the probability of outage is significantly reduced. Indeed, the bigger the value of $D$, the closer the outage probability curve becomes to the black curve which represents the outage probability in the case when no overall interference from the femto network is observed at the tagged macro user due to the perfect sensing ability of the CR femto-BSs. However, the reduction in the outage probability can be less when the number of macroBSs transmitting on the same RB as the tagged macro is larger (see Figure 8b).

For a commercial network, designers must find a way to achieve a lower probability of outage for a certain SINR as the minimum quality needed for a typical femto or macro user to experience an acceptable QoS. A common way to decrease the outage probability is to reduce the number of interfering BSs encountered at the users. This can be done through applying both the (a) and (b) conditions in the perfect sensing scenario (or both the (c) and (d) in the imperfect sensing scenario). As shown in Figure 9, under these constraints, the outage

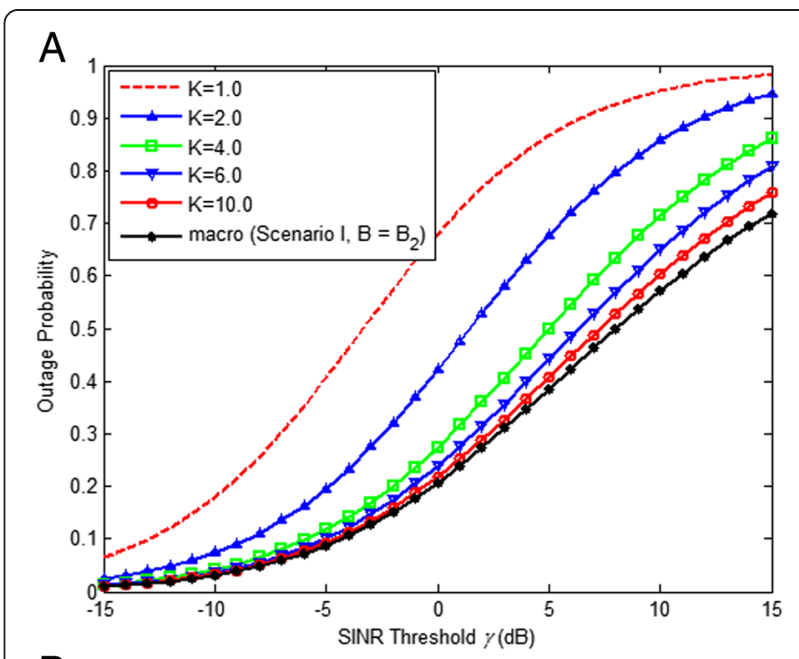

B

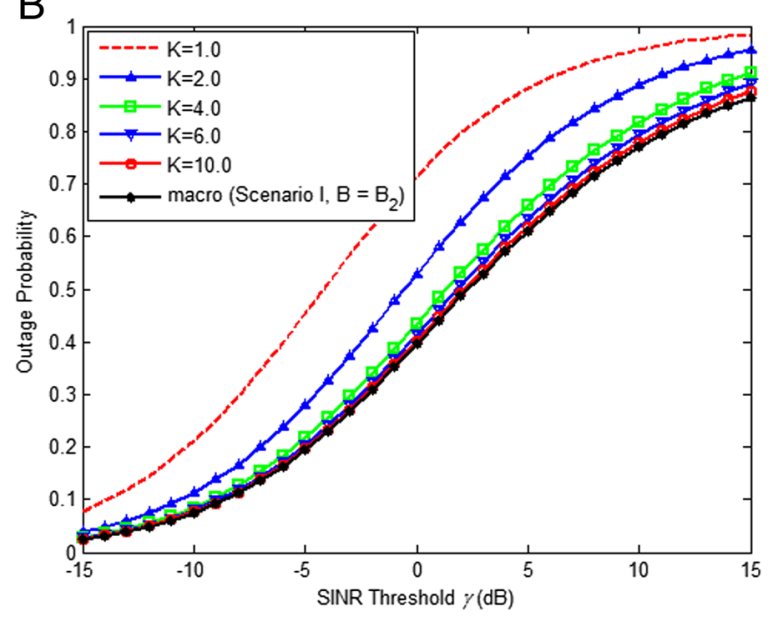

Figure 8 Effect of $D\left(D=\mathrm{Kr}_{M}\right.$, the exclusion region around the tagged macro user) on outage probability of the tagged macro user. The system parameters are set as $P_{F}=23 \mathrm{dBm}, P_{M}=43 \mathrm{dBm}$, $a=4, m_{d, M}=m_{l, M}=m_{l, F}=1, p_{R B}=0.8, p_{t x}=0.7, \lambda_{M}=50, \lambda_{M}^{\prime}=10 \mathrm{in}$ (A) and $\lambda_{M}^{\prime}=25$ in (B).

probability is significantly reduced at the tagged femto user (note that the number of interfering macro-BSs at the tagged femto user is considered equal for all the curves in the figure). The goal is to see the effect of both $p_{R B}$ and $p_{t x}$ (these two parameters are employed at each CR femto-BS) on the outage probability of the tagged femto user. As can be seen, for the case when $p_{R B}=1$ and $p_{t x}=1$, outage occurs with higher probability. In other words, when all the existing femto-BSs (except those who are inside the macro users' exclusion regions) pick the same $\mathrm{RB}$ as the tagged femto $\left(p_{R B}=1\right)$ and when they all have data to transmit in the current time slot $\left(p_{t x}=1\right)$, the tagged femto user will experience the maximum value for the outage probability derived for different SINR targets. Clearly, a significant reduction in the outage probability is occurred for the smaller values of $p_{R B}$ and $p_{t x}$ (see Figure 9) (the smaller the values of 


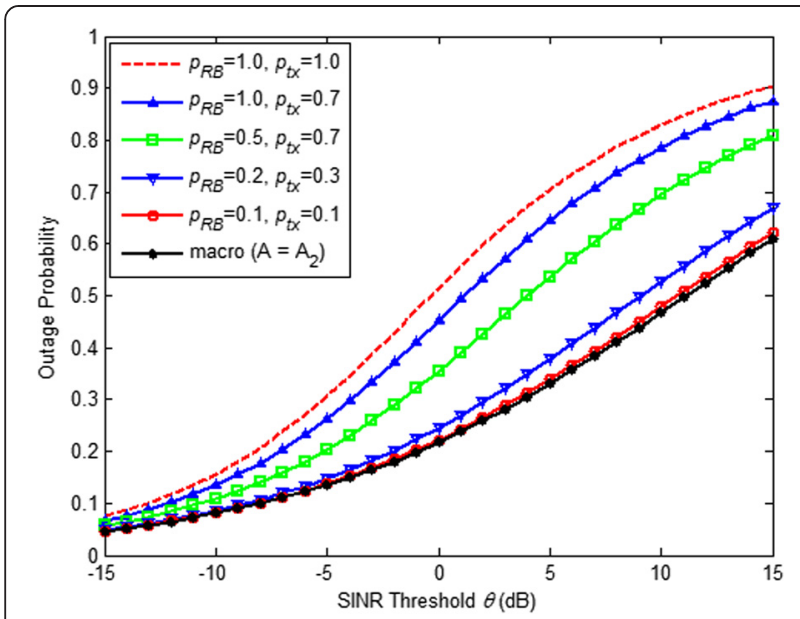

Figure 9 Effect of $p_{R B}$ and $p_{t x}$ (defined parameters for CR femto-BSs) on outage probability of the tagged femto user. The system parameters are set as $P_{F}=23 \mathrm{dBm}, P_{M}=43 \mathrm{dBm}, a=4$, $m_{d, F}=m_{l, F}=m_{I, M}=1, K=1, \lambda_{F}=800, \lambda_{F}^{\prime}=700$ and $\lambda_{M}^{\prime}=10$.

$p_{R B}$ and $p_{t x}$, the closer the outage probability curve becomes to the black curve which represents the outage probability in the case when the received interference at the tagged femto user is only the aggregate interference from the macro network). Indeed, this validates that many studies which do not consider these constraints over-estimate the interference encountered by a typical femto user.

It can be seen that even for high values of $p_{t x}$, the outage probability is relatively less than that in the case where there is no constraint on the femto-BS's transmission schedule $\left(p_{t x}=1\right)$. Also, it is obvious that in the presence of multiple RBs where each RB is picked with probability $p_{R B}$, the outage probability is further decreased. Hence, any practical heterogeneous network designed to satisfy both of the mentioned conditions can reap the benefit of opportunistic exploitation of spectrum, while possibly causing little or no harmful interference. The same story exists when the outage probability of the tagged macro user is investigated. Figure 10 shows the effect of $p_{R B}$ and $p_{t x}$ (parameters related to the CR femto-BSs) on the outage probability of the tagged macro user. For instance, when all the active femto-BSs select the same RB as the tagged macro $\left(p_{R B}=1\right)$ and when they all have data to transmit (on this busy $\mathrm{RB}$ ) in the current time slot $\left(p_{t x}=1\right)$, the tagged macro user will experience the maximum value for the outage probability. It should be noted that the number of interfering macro-BSs at the tagged macro is considered equal for all curves in this figure.

Figure 11 plots the outage probability versus the SINR threshold $\theta$ for the tagged femto user. As $m_{d, F}=m_{I, F}$ increases, the outage probability of the tagged femto user decreases. This is because as the fading becomes less

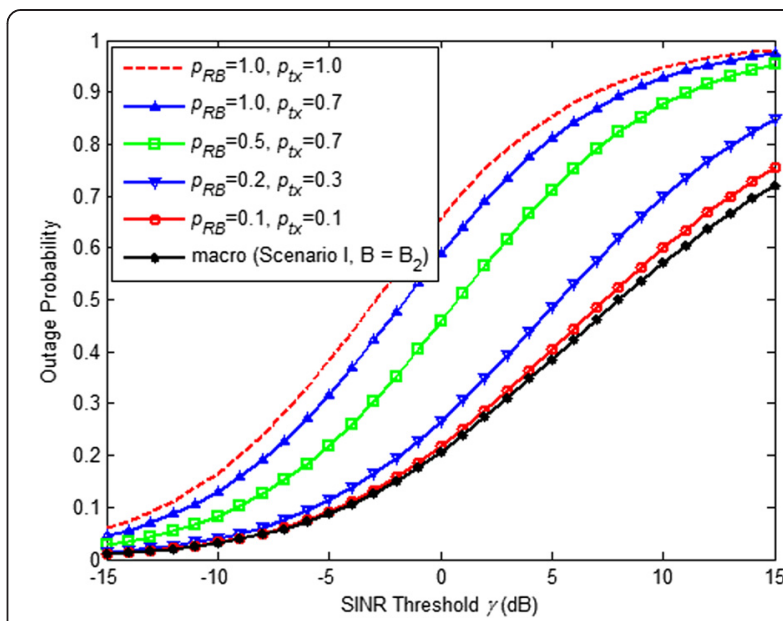

Figure 10 Effect of $p_{R B}$ and $p_{t x}$ (defined parameters for CR femto-BSs) on outage probability of the tagged macro user. The system parameters are set as $P_{F}=23 \mathrm{dBm}, P_{M}=43 \mathrm{dBm}, a=4$, $m_{d, F}=m_{l, F}=m_{l, M}=1, K=1, \lambda_{F}^{\prime}=700, \lambda_{M}=50$ and $\lambda_{M}^{\prime}=10$.

severe (the bigger the Nakagami-m parameter, the smaller fading) for both the desired and interfering links of the femto network, the received power at the tagged femto user from its corresponding femto-BS increases more compared to the total received power from all the interfering femto-BSs. It should be noted that $\beta_{F}=\frac{\Omega_{d, F}}{m_{d, F}}=$ $\frac{\Omega_{l, F}}{m_{l, F}}$ and $\beta_{M}=\frac{\Omega_{d, M}}{m_{d, M}}=\frac{\Omega_{l, M}}{m_{I, M}}$ (the scale parameters) are fixed for all the curves. The interference links between the interfering macro-BSs and the tagged femto user are more likely to be severe in terms of fading compared to the femto network's desired and interfering links. In other words, the interfering signals received at the

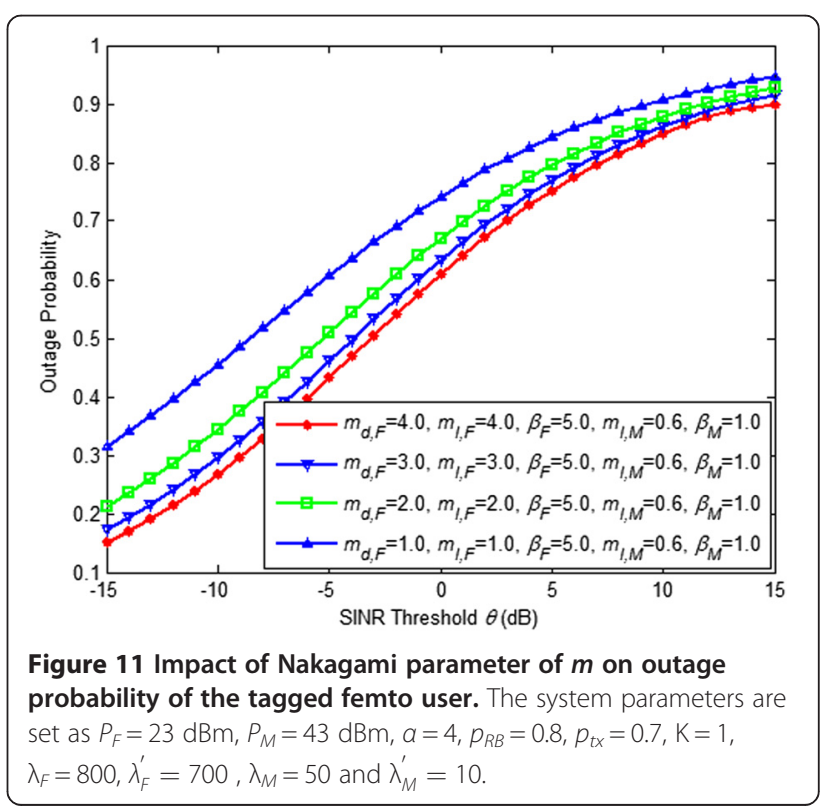


tagged femto user originated by the macro-BSs suffer more serious fading than the received signals from the femto-BSs, in particular when we talk about indoor femto users. Similar arguments and statements can also be made about the outage probability of the tagged macro user observed in Figure 12.

In Figures 13 and 14, we analyze the performance of the authorized links (femto links) in terms of throughput (achievable with a simple ARQ scheme with error-free feedback) under both the perfect and imperfect sensing scenarios. Considering the slotted ALOHA scheme, we define the following terms $[19,20]$,

Probabilistic link throughput $(\tau)$ of a femto user:

(i) In the half-duplex (HD) communication scenario: it is defined to be the success probability of a femto user (i.e., $1-p_{O F}$ ) multiplied by the probability that the corresponding femto-BS actually transmits over a specific RB (i.e., $p_{R B} p_{t x}$ ), and the probability that the femto receiver actually receives over that $\mathrm{RB}$ (i.e., $1-p_{R B} p_{t x}$ )

(ii) In the full-duplex (FD) communication scenario: it is defined to be the success probability of a femto user (i.e., $1-p_{O F}$ ) multiplied by the probability that the corresponding femto-BS actually transmits over a specific RB (i.e., $\left.p_{R B} p_{t x}\right)$.

\section{Femto link throughput (T):}

The femto link throughput is defined as the product of the probabilistic link throughput $(\tau)$ and the rate of transmission, i.e., $T=\tau \log (1+\theta)$. Therefore, the femto link throughput for the half and full duplex cases is written as follows

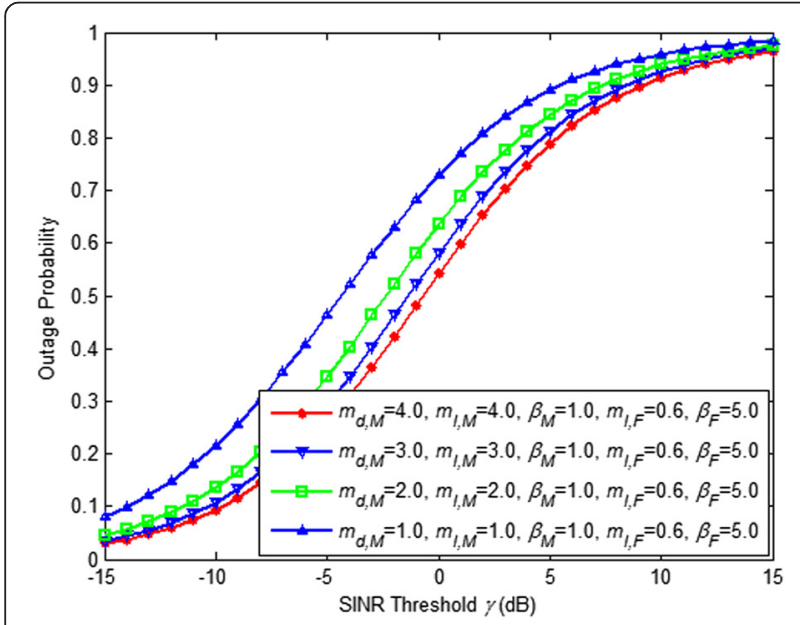

Figure 12 Impact of Nakagami parameter of $m$ on outage probability of the tagged macro user. The system parameters are set as $P_{F}=23 \mathrm{dBm}, P_{M}=43 \mathrm{dBm}, a=4, p_{R B}=0.8, p_{t x}=0.7, \mathrm{~K}=1$, $\lambda_{F}=800, \lambda_{F}^{\prime}=700, \lambda_{M}=50$ and $\lambda_{M}^{\prime}=10$.

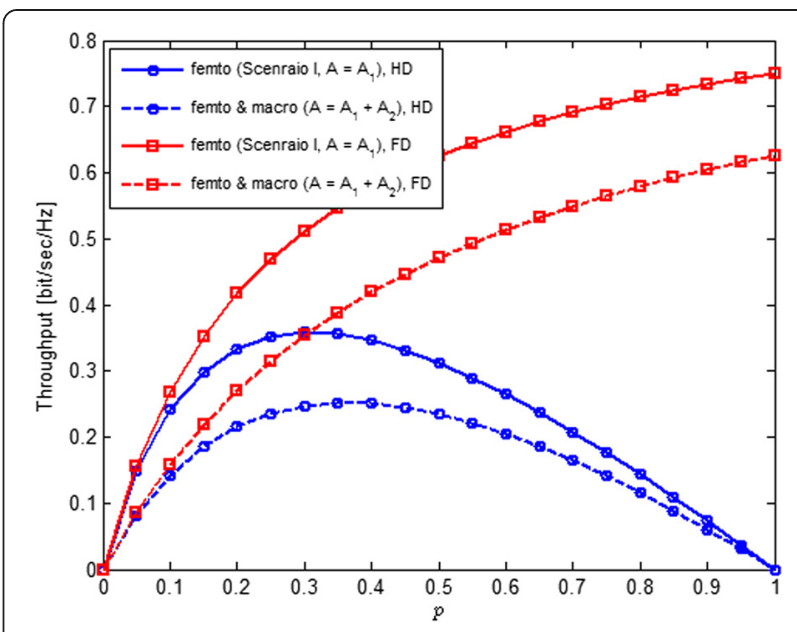

Figure 13 Femto link throughput of half and full duplex systems as a function of transmission probability over a specific RB ( $\boldsymbol{p})$. The system parameters are set as $P_{F}=23 \mathrm{dBm}$, $P_{M}=43 \mathrm{dBm}, a=4, m_{d, F}=m_{l, F}=m_{l, M}=1, K=1, \lambda_{M}^{\prime}=10, \lambda_{F}=800$ and $\lambda_{F}^{\prime}=700$, and $\theta=11 \mathrm{~dB}$.

$$
\begin{gathered}
T^{(\text {half })}=p(1-p)\left(1-p_{O F}\right) \log (1+\theta) \\
T^{(f u l l)}=p\left(1-p_{O F}\right) \log (1+\theta)
\end{gathered}
$$

in which $p=p_{R B} p_{t x}$.

In Figure 13, the performance of half and full duplex systems are presented for the femto users. More specifically, the link throughput of any typical femto user (e.g. the link between the tagged femto user and its corresponding femto-BS) under perfect and imperfect spectrum sensing abilities for the $\mathrm{CR}$ femto-BSs is shown as a function of the transmission probability over a specific RB (i.e., $p=p_{R B} p_{t x}$ ). It can be seen that the

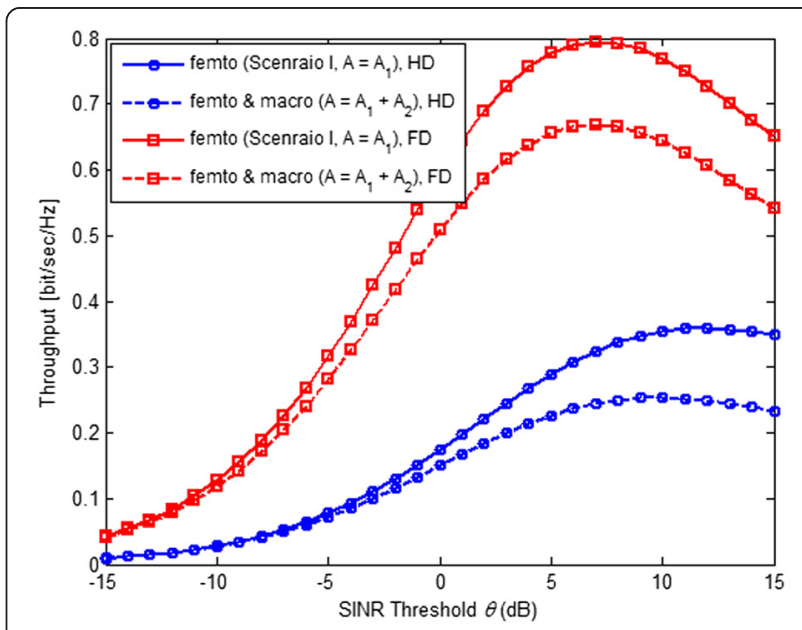

Figure 14 The femto link throughput of half and full duplex systems as a function of the target SINR. The system parameters are set as $P_{F}=23 \mathrm{dBm}, P_{M}=43 \mathrm{dBm}, a=4, m_{d, F}=m_{l, F}=m_{l, M}=1$, $K=1, \lambda_{M}^{\prime}=10, \lambda_{F}=800$ and $\lambda_{F}^{\prime}=700$, and for each curve the obtained optimal $p$ in Figure 13 is applied. 
throughput achieved by the FD system is significantly higher, particularly when $p$ is high. Regarding the performance of the HD system, for both the perfect and imperfect sensing cases, there is a unique optimal $p$ which achieves the maximum throughput ( $p=0.3$ for the perfect and $p=0.35$ for the imperfect sensing scenario). However, for high $p$, both throughput curves converge to zero due to over many transmissions and interferences on the RB. Obviously, for both the half and full duplex communications, a higher per-link throughput is achieved when the CR femto-BSs employ perfect sensing.

In Figure 14, the performance of half and full duplex systems are presented for femto users. More specifically, the link throughput of any typical femto user (e.g., the link between the tagged femto user and its corresponding femto-BS) under perfect and imperfect spectrum sensing abilities for the CR femto-BSs is shown as a function of the target SINR $\theta$. It can be seen that the per-link throughput achieved by the FD system, for both the perfect and imperfect sensing scenarios, is significantly higher than the HD one. As it is seen, the link throughput curves are concave and there is an optimal point in each curve. With a high target SINR, we can transmit the user data with high spectral efficiency; however, the outage probability of this transmission is high, too. In contrast, with a low-target SINR, we can send many packets that include little information. In other words, a high reliable transmission can be experienced at low-target SINRs, while the minimum requirements for the transmission rate cannot be met.

\section{Conclusion}

In this paper, utilizing the spatial PPP theory, we presented a tractable model to derive the outage probability of a typical femto and macro user in a two-tier heterogeneous network which provides insight into system design guidelines. In other words, for the case of the node locations modeled by a PPP and Nakagami-m fading channels, we demonstrated the use of the CR-based framework to evaluate the outage probability at any arbitrary user. Exact closed-form expressions were obtained as a result. In addition, we observed that in the downlink analysis, the outage probability is a function of the network topology and several important system design parameters such as SINR target, exclusion regions, MAC (medium access control) mechanisms such as ALOHA $\left(p_{t x}\right)$, and the RB selection constraint $\left(p_{R B}\right)$ which is controlled by the spectrum sensing measurements.

\section{Appendix 1}

7.1 Proof of equation 6

Proof: From (5), we have

$$
\begin{aligned}
P[\operatorname{SINR}>\theta]= & \int_{0}^{\infty}\left[e ^ { - ( \frac { m _ { d , F } \theta _ { r _ { F } } ^ { \alpha } } { \Omega _ { d , F } F _ { F } } ) I } \left[1+\left(\frac{m_{d, F}}{\Omega_{d, F}} \frac{\theta r_{F}^{\alpha}}{P_{F}}\right) I\right.\right. \\
& \left.\left.+\frac{1}{2}\left(\frac{m_{d, F}}{\Omega_{d, F}} \frac{\theta r_{F}^{\alpha}}{P_{F}}\right)^{2} I^{2}+\ldots\right]\right] f_{I}(i) \mathrm{d} i
\end{aligned}
$$

We then have

$$
\begin{aligned}
& {\left[\left[\left(\frac{m_{d, F}}{\Omega_{d, F}} \frac{\theta r_{F}^{\alpha}}{P_{F}}\right)^{0} \int_{0}^{\infty}\left[e^{-s I}\left(I^{0} f_{I}(i)\right)\right] \mathrm{d} i\right]\right.} \\
& +\left[\left(\frac{m_{d, F}}{\Omega_{d, F}} \frac{\theta r_{F}^{\alpha}}{P_{F}}\right)^{1} \int_{0}^{\infty}\left[e^{-s I}\left(I^{1} f_{I}(i)\right)\right] \mathrm{d} i\right] \\
& \left.+\left[\frac{1}{2}\left(\frac{m_{d, F}}{\Omega_{d, F}} \frac{\theta r_{F}^{\alpha}}{P_{F}}\right)^{2} \int_{0}^{\infty}\left[e^{-s I}\left(I^{2} f_{I}(i)\right)\right] \mathrm{d} i\right]+\ldots\right]
\end{aligned}
$$

where $s$ in each integral is equal to $\frac{m_{d, F}}{\Omega_{d, F}} \frac{\theta r_{F}{ }^{\alpha}}{P_{F}}$.

In each part, we have to calculate the Laplace transform of $\left(I^{n} f_{I}(i)\right)$. We also know that $x^{n} f(x) \stackrel{\mathscr{L}}{\Leftrightarrow}(-1)^{n} \frac{d^{n} \mathscr{L}_{f}(s)}{d s^{n}}$. Therefore, we have

$$
\begin{aligned}
& {\left[\left[\left(\frac{m_{d, F}}{\Omega_{d, F}} \frac{\theta r_{F}{ }^{\alpha}}{P_{F}}\right)^{0}(-1)^{0} \frac{d^{0} \mathscr{L}_{I}(s)}{d s^{0}}\right]\right.} \\
& +\left[\left(\frac{m_{d, F}}{\Omega_{d, F}} \frac{\theta r_{F}^{\alpha}}{P_{F}}\right)^{1}(-1)^{1} \frac{d^{1} \mathscr{L}_{I}(s)}{d s^{1}}\right] \\
& \left.+\left[\frac{1}{2}\left(\frac{m_{d, F}}{\Omega_{d, F}} \frac{\theta r_{F}^{\alpha}}{P_{F}}\right)^{2}(-1)^{2} \frac{d^{2} \mathscr{L}_{I}(s)}{d s^{2}}\right]+\ldots\right]
\end{aligned}
$$

Hence, we have

$$
P[\operatorname{SINR}>\theta]=\left[\sum_{k=0}^{m_{d, F}-1} \frac{(s)^{k}}{k !}(-1)^{k} \frac{d^{k} \mathscr{L}_{I}(s)}{d s^{k}}\right]
$$

\section{Appendix 2}

\subsection{Proof of equation 15}

Proof: From (14), we have

$$
\begin{aligned}
M(\theta, \alpha)= & \int_{0}^{\infty}\left[( g ) ^ { \frac { d } { \alpha } } \left(\Gamma\left(-\frac{d}{\alpha}, \frac{m_{d, F}}{\Omega_{d, F}} \theta g\right)\right.\right. \\
& \left.\left.-\Gamma\left(-\frac{d}{\alpha}\right)\right)\right] \frac{m_{I, F} m_{I, F}}{\Omega_{I, F} m_{I, F} \Gamma\left(m_{I, F}\right)} g^{m_{I, F}-1} e^{-\frac{m_{I, F}}{\Omega_{I, F}}} \mathrm{~d} g
\end{aligned}
$$

We know that $\Gamma(a, x)+\gamma(a, x)=\Gamma(a)$, and $\gamma(a, x)=$ $x^{a} \Gamma(a) e^{-x} \sum_{k=0}^{\infty} \frac{x^{k}}{\Gamma(a+k+1)}$, therefore, the above equation is simplified as follows 


$$
\begin{aligned}
& =\frac{-\Gamma\left(-\frac{d}{\alpha}\right) m_{I, F} m_{l, F}\left(\frac{m_{d, F}}{\Omega_{d, F}} \theta\right)^{-\frac{d}{\alpha}}}{\Omega_{I, F} m_{l, F} \Gamma\left(m_{l, F}\right)} \int_{0}^{\infty}(g)^{m_{l, F}-1} e^{-g\left(\frac{m_{l, F}}{\Omega_{I, F}}+\frac{m_{d, F} \theta}{\Omega_{d, F}}\right)}\left[\sum_{k=0}^{\infty} \frac{\left(\frac{m_{d, F}}{\Omega_{d, F}} \theta\right)^{k} g^{k}}{\Gamma\left(-\frac{d}{\alpha}+k+1\right)}\right] \mathrm{d} g
\end{aligned}
$$

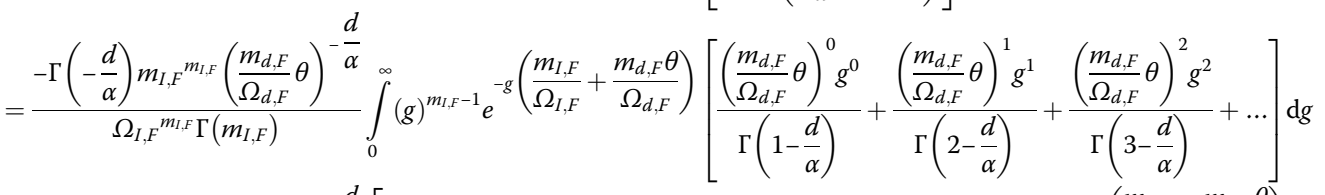

$$
\begin{aligned}
& =\frac{-\Gamma\left(-\frac{d}{\alpha}\right) m_{I, F} m_{l, F}\left(\frac{m_{d, F}}{\Omega_{d, F}} \theta\right)^{-\frac{d}{\alpha}}}{\Omega_{I, F} m_{l, F} \Gamma\left(m_{l, F}\right)}\left[\int_{0}^{\infty} \frac{(g)^{m_{l, F}-1} e^{-g\left(\frac{m_{I, F}}{\Omega_{I, F}}+\frac{m_{d, F} \theta}{\Omega_{d, F}}\right)}}{\Gamma\left(1-\frac{d}{\alpha}\right)} \mathrm{d} g+\int_{0}^{\infty} \frac{\left(\frac{m_{d, F}}{\Omega_{d, F}} \theta\right)^{1}(g)^{\left(m_{l, F}+1\right)-1} e^{-g\left(\frac{m_{I, F}}{\Omega_{I, F}}+\frac{m_{d, F} \theta}{\Omega_{d, F}}\right)}}{\Gamma\left(2-\frac{d}{\alpha}\right)} \mathrm{d} g+\ldots\right] \\
& =\frac{-\Gamma\left(-\frac{d}{\alpha}\right) m_{l, F} m_{l, F}\left(\frac{m_{d, F}}{\Omega_{d, F}} \theta\right)^{-\frac{d}{\alpha}}}{\Omega_{I, F} m_{l, F} \Gamma\left(m_{l, F}\right)}\left[\frac{\left(\frac{m_{d, F}}{\Omega_{d, F}} \theta\right)^{0}\left(\frac{\Omega_{I, F} \Omega_{d, F}}{m_{l, F} \Omega_{d, F}+\Omega_{l, F} m_{d, F} \theta}\right)^{m_{l, F}}}{\Gamma\left(1-\frac{d}{\alpha}\right)} \Gamma\left(m_{l, F}\right)+\frac{\left(\frac{m_{d, F}}{\Omega_{d, F}} \theta\right)^{1}\left(\frac{\Omega_{I, F} \Omega_{d, F}}{m_{l, F} \Omega_{d, F}+\Omega_{l, F} m_{d, F} \theta}\right)^{m_{I, F}+1}}{\Gamma\left(2-\frac{d}{\alpha}\right)} \Gamma\left(m_{l, F}+1\right)+\ldots\right]
\end{aligned}
$$

Hence, we have

$$
M(\theta, \alpha)=\left(\frac{-\Gamma\left(-\frac{d}{\alpha}\right) m_{I, F} m_{I, F}\left(\frac{m_{d, F}}{\Omega_{d, F}} \theta\right)^{-\frac{d}{\alpha}}}{\Omega_{I, F} m_{l, F} \Gamma\left(m_{I, F}\right)}\right) \times\left[\sum_{k=0}^{\infty} \frac{\left(\frac{m_{d, F}}{\Omega_{d, F}} \theta\right)^{k}\left(\frac{\Omega_{I, F} \Omega_{d, F}}{m_{l, F} \Omega_{d, F}+\Omega_{I, F} m_{d, F} \theta}\right)^{m_{l, F}+k}}{\Gamma\left(k+1-\frac{d}{\alpha}\right)} \Gamma\left(m_{I, F}+k\right)\right]
$$

\section{Appendix 3}

\subsection{Outage probability expression for non-integer $m_{d, F}$} Proof: From Section 3

For non-integer $m_{d, F}$ by using the infinite series representation of incomplete gamma function presented in Appendix $2\left(\frac{\Gamma(a, x)}{\Gamma(a)}=1-\frac{\gamma(a, x)}{\Gamma(a)}\right.$, and $\gamma(a, x)=x^{a} \Gamma(a) e^{-x}$ $\left.\sum_{k=0}^{\infty} \frac{x^{k}}{\Gamma(a+k+1)}\right)$, and following the exact procedure as that in Section 3 (from Eq. (1) to Eq. (21)) to obtain the closed-form outage probability expression for the tagged femto user, we obtain an exact but infinite summation expression for the outage probability. In other words, we use the infinite series representation of incomplete gamma not only for the interfering links calculations but also for the desired link to obtain an expression in which the Nakagami-m parameters of all the communication links $\left(m_{d, F}, m_{I, F}\right.$ and $\left.m_{I, M}\right)$ can take any values (integer or non-integer) bigger than 0.5. Thus, considering the above explanations we have

$$
p_{O F}=\sum_{k=0}^{\infty} \frac{(z)^{k+m_{d, F}}}{\Gamma\left(k+m_{d, F}+1\right)}(-1)^{k+m_{d, F}} \frac{d^{k+m_{d, F}}(\exp (l(z)))}{d z^{k+m_{d, F}}}
$$

where

$$
\exp (l(z))=e^{[\mathrm{A}] z^{\frac{d}{\alpha}-\frac{m_{d, F} \theta^{2}}{\Omega_{d, F} F_{F}}} z} \text {, and } z=r_{F}^{\alpha} .
$$

Operating in an interference-limited regime and using the Taylor series expansions of exponential functions, the above expression is simplified as follows

$$
\begin{aligned}
& \left.p_{O F}=\sum_{k=0}^{\infty} \frac{(z)^{k+m_{d, F}}}{\Gamma\left(k+m_{d, F}+1\right)}(-1)^{k+m_{d, F}} \frac{d^{k+m_{d, F}}\left(\sum_{n=0}^{\infty} \frac{\mathrm{A}^{n} z}{n !}\right)}{d z^{k+m_{d, F}}\left(\frac{d}{\alpha}\right)}\right) \\
& p_{O F}=\sum_{k=0}^{\infty} \frac{(z)^{k+m_{d, F}}}{\Gamma\left(k+m_{d, F}+1\right)}(-1)^{k+m_{d, F}} \frac{d^{k+m_{d, F}}\left(\frac{\mathrm{A}^{0} z^{0}}{0 !}+\frac{\mathrm{A}^{1} z}{\left(\frac{d}{\alpha}\right)}+\frac{\mathrm{A}^{2} z^{2}\left(\frac{d}{\alpha}\right)}{2 !}+\ldots\right)}{d z^{k+m_{d, F}}}
\end{aligned}
$$

The fractional derivative, the arbitrary order derivative, is used here to simplify the above expression [21] (the notation $D^{\mu}(f(z))=\frac{d^{\mu}(f(z))}{d z^{\mu}}$ denotes the $\mu$ th order fractional derivative of the function $f(z)$ where $\mu>0)$. According to [21], $D^{\mu}\left(z^{\gamma}\right)$ (where $\gamma>-1$ ) is equal to $\frac{\Gamma(\gamma+1)}{\Gamma(\gamma-\mu+1)} z^{\gamma-\mu}$, and we also have $D^{\mu}(c f(z))=c D^{\mu}(f(z))(c$ is a constant value). Thus, we can write 


$$
\begin{aligned}
p_{O F}= & \sum_{k=0}^{\infty} \frac{(z)^{k+m_{d, F}}}{\Gamma\left(k+m_{d, F}+1\right)}(-1)^{k+m_{d, F}}\left[\frac{\mathrm{A}^{0} \Gamma\left(0\left(\frac{d}{\alpha}\right)+1\right) z^{0\left(\frac{d}{\alpha}\right)-\left(k+m_{d, F}\right)}}{0 ! \Gamma\left(0\left(\frac{d}{\alpha}\right)-\left(k+m_{d, F}\right)+1\right)}+\frac{\mathrm{A}^{1} \Gamma\left(1\left(\frac{d}{\alpha}\right)+1\right) z^{1\left(\frac{d}{\alpha}\right)-\left(k+m_{d, F}\right)}}{1 ! \Gamma\left(1\left(\frac{d}{\alpha}\right)-\left(k+m_{d, F}\right)+1\right)}\right. \\
& \left.+\frac{\mathrm{A}^{2} \Gamma\left(2\left(\frac{d}{\alpha}\right)+1\right) z^{2\left(\frac{d}{\alpha}\right)-\left(k+m_{d, F}\right)}}{2 ! \Gamma\left(2\left(\frac{d}{\alpha}\right)-\left(k+m_{d, F}\right)+1\right)}+\ldots\right]
\end{aligned}
$$

and finally,

$$
p_{O F}=\sum_{k=0}^{\infty} \frac{(z)^{k+m_{d, F}}}{\Gamma\left(k+m_{d, F}+1\right)}(-1)^{k+m_{d, F}} \sum_{n=0}^{\infty} \frac{\mathrm{A}^{n}}{n !} \frac{\Gamma\left(n\left(\frac{d}{\alpha}\right)+1\right)}{\Gamma\left(n\left(\frac{d}{\alpha}\right)-\left(k+m_{d, F}\right)+1\right)} z^{n\left(\frac{d}{\alpha}\right)-\left(k+m_{d, F}\right)}
$$

where A is given by (22).

Similar expression can be obtained for the outage probability of the tagged macro user.

\section{Competing interests}

The authors declare that they have no competing interests.

Received: 8 August 2014 Accepted: 9 February 2015

Published online: 10 March 2015

\section{References}

1. J Andrews, H Claussen, M Dohler, S Rangan, M Reed, Femtocells: past, present, and future. IEEE J on Sel Areas in Comm 30(3), 497-508 (2012)

2. S Cheng, S Lien, $\mathrm{F} \mathrm{Hu}, \mathrm{K}$ Chen, On exploiting cognitive radio to mitigate interference in macro/femto heterogeneous networks. IEEE Wireless Commun Mag 18(3), 40-47 (2011)

3. H ElSawy, E Hossain, M Haenggi, Stochastic geometry for modeling, analysis, and design of multi-tier and cognitive cellular wireless networks: a survey. IEEE Commun Surveys \& Tutorials 15(3), 996-1019 (2013)

4. K Gilhousen, I Jacobs, R Padovani, AJ Viterbi, L Weaver, C Wheatley, On the capacity of a cellular CDMA system. IEEE Trans on Veh Technology 40(2), 303-312 (1991)

5. J Xu, J Zhang, JG Andrews, On the accuracy of the Wyner model in cellular networks. IEEE Trans on Wireless Commun 10(9), 3098-3109 (2011)

6. JG Andrews, F Baccelli, RK Ganti, A tractable approach to coverage and rate in cellular networks. IEEE Trans on Commun 59(11), 3122-3134 (2011)

7. SAR Zaidi, DC McLernon, M Ghogho, Outage probability analysis of cognitive radio networks under self-coexistence constraint. (44th Annual Conf. on Inf. Sciences and Systems (CISS), 2010), pp.1. 6, 17-19

8. RK Ganti, M Haenggi, Interference and outage in clustered wireless ad hoc networks. IEEE Trans on Inf Theory 55(9), 4067-4086 (2009)

9. A Hasan, JG Andrews, The guard zone in wireless ad hoc networks. IEEE Trans on Wireless Commun 4(3), 897-906 (2007)

10. C-H Lee, M Haenggi, Interference and outage in Poisson cognitive networks. IEEE Trans on Wireless Commun 11(4), 1392-1401 (2012)

11. M Haenggi, RK Gant, Interference in large wireless networks. Foundations and Trends in Networking 3(2), 127-248 (2008). NOW Publisher

12. IEEE Instrumentation and Measurement Society, IEEE 1588 standard for a precision clock synchronization protocol for networked measurement and control systems. IEEE Std 1588, (2008)

13. Q Chen, M Motani, WC Wong, YC Liang, Opportunistic spectrum access protocol for cognitive radio networks. in Proc. IEEE International Conf. on Commun. (ICC). (2011), pp. 1-6

14. X Li, H Liu, S Roy, J Zhang, P Zhang, C Ghosh, Throughput analysis for a multi-user, multi-channel ALOHA cognitive radio system. IEEE Trans on Wireless Commun 11(11), 3900-3909 (2012)

15. Y-C Liang, Y Zeng, E Peh, AT Hoang, Sensing-throughput tradeoff for cognitive radio networks. IEEE Trans on Wireless Commun 7(4), 1326-1337 (2008)

16. L Luo, C Ghosh, S Roy, Joint optimization of spectrum sensing for cognitive radio networks. in Proc. IEEE Global Telecommun. Conf. (GLOBECOM), (2010), pp. 1-5

17. Q Zhao, B Sadler, A survey of dynamic spectrum access: signal processing, networking, and regulatory policy. IEEE Sig Proc Magazine 24(3), 79-89 (2007)
18. D Kim, On upper bounds of SIR-based call admission threshold in power-controlled DS-CDMA mobile systems. IEEE Commun Letters 6(1), 13-15 (2002)

19. M Haenggi, Outage, local throughput, and capacity of random wireless networks. IEEE Trans on Wireless Commun 8(8), 4350-4359 (2009)

20. J-M Dricot, G Ferrari, A Panahandeh, Fr Horlin, Ph. De Doncker, Probabilistic coexistence and throughput of cognitive dual-polarized networks. EURASIP Journal on Wireless Commun. and Networking, 2010:387625.

21. AA Kilbas, HM Srivastava, JJ Trujillo, Theory and applications of fractional differential equations (Elsevier B.V, Netherlands, 2006)

\section{Submit your manuscript to a SpringerOpen ${ }^{\circ}$ journal and benefit from:}

- Convenient online submission

- Rigorous peer review

- Immediate publication on acceptance

- Open access: articles freely available online

- High visibility within the field

- Retaining the copyright to your article

Submit your next manuscript at $>$ springeropen.com 